\title{
SQUARE-FREE VALUES OF DECOMPOSABLE FORMS
}

\author{
STANLEY YAO XIAO
}

\begin{abstract}
In this paper we prove that decomposable forms, or homogeneous polynomials $F\left(x_{1}, \cdots, x_{n}\right)$ with integer coefficients which split completely into linear factors over $\mathbb{C}$, take on infinitely many square-free values subject to simple necessary conditions and $\operatorname{deg} f \leq 2 n+2$ for all irreducible factors $f$ of $F$. This work generalizes a theorem of Greaves.
\end{abstract}

\section{INTRODUCTION}

In this paper, we consider the density of integer tuples $\left(x_{1}, \cdots, x_{n}\right)$ satisfying $\left|x_{i}\right| \leq B$ and for which $F\left(x_{1}, \cdots, x_{n}\right)$ is square-free, where $F$ is an $n$-ary decomposable form of degree $d>n$. A homogeneous polynomial $F$ is said to be a decomposable form if it splits into linear factors over the algebraic closure of its field of definition. If $F$ has rational coefficients and is irreducible over $\mathbb{Q}$, we say that $F$ is an incomplete norm form. Before stating our result, we shall give a brief summary of work done on square-free values of polynomials to date.

For a polynomial $g(x)$ with integer coefficients, define the counting function

$$
N_{g}(B)=\#\{x \in \mathbb{Z}:|x| \leq B, g(x) \text { is square-free }\} .
$$

Estermann [10] showed that when $g(x)=x^{2}+1$, there exists a positive number $c_{g}$ such that the asymptotic formula

$$
N_{g}(B)=c_{g} B+O\left(B^{2 / 3} \log B\right)
$$

holds. We will say that a polynomial $g$ has no fixed square divisor if for all primes $p$ there exists $n_{p} \in \mathbb{Z}$ such that $p^{2} \nmid g\left(n_{p}\right)$. Ricci [34] generalized Estermann's work and showed that for any irreducible quadratic polynomial with no fixed square divisor, there exists a positive number $c_{g}$ such that (1.1) holds. Erdös showed that

$$
\lim _{B \rightarrow \infty} N_{g}(B)=\infty
$$

in [8] for cubic polynomials with no fixed square divisor. Hooley [24] refined the work of Estermann, Ricci, and Erdős and showed that for all cubic polynomials $g$ with no fixed square divisor, there exists a positive number $c_{g}$ such that (1.1) holds with a worse error term. Helfgott further refined Hooley's work in [22] by showing that an analogous asymptotic formula to (1.1) holds when we replace integer inputs with prime inputs. To date, it is not known whether (1.1) holds unconditionally for any polynomial $g$ with no fixed square divisor with $\operatorname{deg} g \geq 4$.

Date: October 3, 2018.

2010 Mathematics Subject Classification. Primary 11B05.

Key words and phrases. Power-free values, polynomials, number theory. 
Assuming the abc-conjecture, Granville and Poonen proved respectively in [14] and 32 that polynomials in a single variable and polynomials in multiple variables take on infinitely many square-free values. We note that Poonen's result does not allow one to deduce an analogous asymptotic formula to (1.1). Bhargava, Shankar, and Wang recently showed the existence of an asymptotic formula for square-free values of discriminant polynomials, which does not use the abc-conjecture in [3].

A natural generalization from the case of single-variable polynomials is to binary forms. Greaves made a breakthrough in [15] on the problem of square-free values of binary forms for suitable binary forms $F(x, y)$ with integer coefficients with no fixed square divisor. He showed that the density of integer pairs $(x, y)$ such that $F(x, y)$ is square-free is exactly as expected provided that $d^{\prime} \leq 6$, where $d^{\prime}$ is the largest degree of an irreducible factor of $F$. One observes that the requirement $d^{\prime} \leq 6$ can be compared to $d \leq 3$ in the single variable case. Hooley, in [25] and [26], extended Greaves's results to the case when $F$ is a polynomial in two variables which splits into linear factors over $\mathbb{C}$.

Schmidt, in [36, introduced an invariant which he called the discriminant for (incomplete) norm forms which we define below. Write

$$
F(\mathbf{x})=\prod_{j=1}^{d} L_{j}(\mathbf{x})
$$

where the $L_{j}$ 's are conjugates of the linear form

$$
L_{1}(\mathbf{x})=\omega_{1} x_{1}+\omega_{2} x_{2}+\cdots+\omega_{n} x_{n}
$$

with algebraic integer coefficients in a number field $K$. We then put

$$
\Delta(F)=\prod_{\left\{i_{1}, \cdots, i_{n}\right\} \subset\{1, \cdots, d\}}\left|\operatorname{det}\left(L_{i_{1}}, \cdots, L_{i_{n}}\right)\right|,
$$

where the determinant of $n$ linear forms in $x_{1}, \cdots, x_{n}$ refers to the determinant of its coefficients. It is easy to check that $\Delta(F)$ is invariant under any action of the Galois group $\operatorname{Gal}(\overline{\mathbb{Q}} / \mathbb{Q})$, and since each term that appears in the product is an algebraic integer, it follows that $\Delta(F)$ is a rational integer. We say that $F$ has bad reduction at a prime $p$ if $F$ has a repeated linear factor over $\mathbb{F}_{p}$. One notes that bad reduction can only occur if $p \mid \Delta(F)$. Therefore, if $\Delta(F)$ is non-zero, then bad reduction can only occur at finitely many primes.

In this paper, we extend Greaves's work in [15] and Hooley's work in [25] and [26] by generalizing Greaves's geometry of numbers method for $n$-ary decomposable forms and adapting Hooley's sieve arguments.

For an integer $k$ and an integer $m$, we say that $m$ is $k$-free if for all primes $p$ dividing $m$, we have $p^{k} \nmid m$. For a set $S$, we write $\# S$ for the cardinality of $S$. Let us write, for an $n$-ary form $F$ with integer coefficients,

$$
\rho_{F}(m)=\#\left\{\left(a_{1}, \cdots, a_{n}\right) \in(\mathbb{Z} / m \mathbb{Z})^{n}: F\left(a_{1}, \cdots, a_{n}\right) \equiv 0 \quad(\bmod m)\right\}
$$


and for a positive number $B$ and an integer $k \geq 2$,

$$
N_{F, k}(B)=\#\left\{\left(x_{1}, \cdots, x_{n}\right) \in \mathbb{Z}^{n}:\left|x_{i}\right| \leq B, F\left(x_{1}, \cdots, x_{n}\right) \text { is } k \text {-free }\right\} .
$$

We will prove the following theorem:

Theorem 1.1. Write $\mathbf{x}=\left(x_{1}, \cdots, x_{n}\right)$ and let

$$
F(\mathbf{x})=L_{1}(\mathbf{x}) \cdots L_{r}(\mathbf{x})
$$

be a decomposable form with integer coefficients and non-zero discriminant $\Delta(F)$ as given in (1.3), where $L_{1}, \cdots, L_{r}$ are linear forms with algebraic integral coefficients in some finite extension $K / \mathbb{Q}$. Let $d$ be the maximal degree of a $\mathbb{Q}$-irreducible factor of $F$. Let $k \geq 2$ be an integer with the property that for all primes $p$, there exists a vector $\mathbf{x}^{(p)}=\left(x_{1}^{(p)}, \cdots, x_{n}^{(p)}\right) \in \mathbb{Z}^{n}$ such that $p^{k} \nmid F\left(\mathbf{x}^{(p)}\right)$. Then the asymptotic relation

$$
N_{F, k}(B) \sim B^{n} \prod_{p}\left(1-\frac{\rho_{F}\left(p^{k}\right)}{p^{n k}}\right)
$$

holds whenever

$$
k \geq \frac{d-2}{n} .
$$

In particular, if $k=2$, then $F$ takes on infinitely many square-free values as long as $d \leq 2 n+2$. This recovers the theorem of Greaves in [15]. We further remark that J. Maynard, in [29], used methods from geometry of numbers related to the methods in Section 3 , to prove an analogous theorem to Theorem 1.1 for primes represented by incomplete norm forms.

The outline of our paper is as follows. In Section 2 we will use an elementary sieve argument to partition the relevant main terms and error terms to be estimated in order to prove Theorem 1.1, In Section 3, we will generalize Greaves's geometry of numbers argument in [15] to the case of decomposable forms over $\mathbb{Z}$. In Sections 4 and 5, we adapt the Ekedahl Sieve as described in [2] and [7] and the Selberg sieve, as expressed by Hooley in [25], to establish an estimate for the remaining error terms relevant to condition (1.6) of Theorem 1.1 .

Funding. This work was supported by the Government of Ontario; University of Waterloo; and the Natural Sciences and Engineering Research Council of Canada.

Acknowledgments. The author would like to thank C.L. Stewart and D. Tweedle for their comments on the paper and discussions.

\section{Preliminaries}

We will show that $N_{F, k}(B)$ (recall (1.5)) satisfies an inequality of the form

$$
N_{1}(B)-N_{2}(B)-N_{3}(B) \leq N_{F, k}(B) \leq N_{1}(B) .
$$

Our goal will be to demonstrate that for any $\varepsilon>0$, that

$$
N_{1}(B)=B^{n} \prod_{p \leq \xi_{1}}\left(1-\frac{\rho_{F}\left(p^{k}\right)}{p^{n k}}\right)+O_{F, \varepsilon}\left(B^{n-1+\varepsilon}\right)
$$


and for some $\delta_{n}>0$ and some slowly growing function $\xi_{1}=\xi_{1}(B)$ tending to infinity as the parameter $B$ tends to infinity, that

$$
N_{2}(B)=O_{F}\left(B^{n}\left(\xi_{1}^{-1}+(\log B)^{-\delta_{n}}\right)\right)
$$

and that

$$
N_{3}(B)=o_{F}\left(B^{n}\right) \text {. }
$$

Put $\log _{1}(B)=\max \{1, \log B\}$ and $\log _{s} B=\log _{1} \log _{s-1} B$ for $s \geq 2$. We now write

$$
\xi_{1}=\xi_{1}(B),
$$

to be an eventually increasing real-valued function tending to infinity which we shall define later. For now, it suffices to suppose that $\xi_{1}(B)=O\left(\log _{2} B / \log _{3} B\right)$. Next put

$$
\xi_{2}=B^{n}(\log B)^{2 / 3} \text {. }
$$

Now define

$$
\begin{gathered}
N_{2}(B)=\#\left\{\mathbf{x} \in \mathbb{Z}^{n}:\left|x_{i}\right| \leq B \text {, there exists } p \in\left(\xi_{1}, \xi_{2}\right] \text { s.t. } p^{2} \mid F(\mathbf{x}),\right. \text { and } \\
\text { if } \left.p^{k} \mid F(\mathbf{x}) \text {, then } p>\xi_{1}\right\},
\end{gathered}
$$

and

$N_{3}(B)=\#\left\{\mathbf{x} \in \mathbb{Z}^{n}:\left|x_{i}\right| \leq B\right.$, there exists $p>\xi_{2}$ s.t. $p^{k} \mid F(\mathbf{x}), F(\mathbf{x})$ is indivisible by

$$
\left.p^{2} \text { for } \xi_{1}<p \leq \xi_{2} \text { and if } p^{k} \mid F(\mathbf{x}) \text {, then } p>\xi_{1}\right\} \text {. }
$$

Before we proceed with estimating $N_{1}(B)$, let us establish some facts about the function $\rho_{F}$ as defined in (1.4). For a positive integer $m$ and a real number $\alpha$, let us write

$$
\sigma_{\alpha}(m)=\sum_{s \mid m} s^{\alpha}
$$

Furthermore, for each prime $p$ we define

$$
\tau_{F}(p)=\# \text { geometrically irreducible components of } F \text { defined over } \mathbb{F}_{p},
$$

and for square-free integers we define

$$
\tau_{F}(m)=\prod_{p \mid m} \tau_{F}(p) .
$$

We remark that in our case, the only geometrically irreducible components are hyperplanes which are defined over $\mathbb{F}_{p}$.

We will establish the following lemma:

Lemma 2.1. Let $\rho_{F}$ be defined as in (1.4). Then $\rho_{F}$ is multiplicative and for all primes $p$ we have

$$
\rho_{F}\left(p^{k}\right)=O_{d, n}\left(p^{k(n-1)}+p^{n(k-1)}\right) .
$$

If $m$ is a square-free integer, then

$$
\rho_{F}(m)=O_{F}\left(m^{n-1} \tau_{F}(m) \sigma_{-1 / 4}(m)\right) .
$$


Proof. The fact that $\rho_{F}$ is multiplicative follows from the Chinese Remainder Theorem. For the upper bound, let us first suppose that there exists an index, say $i=1$, such that $p \nmid x_{1}$. Then there are at most $p^{k}$ many choices for $x_{2}, \cdots, x_{n}$. Having fixed these, there are then at most $d$ choices for $x_{1}$. Hence, there are at most $n d p^{(n-1) k}$ choices for $\left(x_{1}, \cdots, x_{n}\right)$. Otherwise, suppose that $p \mid x_{i}$ for $i=1, \cdots, n$. Write $x_{i}=p x_{i}^{\prime}$ for $i=1, \cdots, n$. Then there are at most $p^{k-1}$ choices for each $i=1, \cdots, n$, whence there are $p^{n(k-1)}$ choices altogether. Combining these, we obtain the claimed upper bound.

For the second part, we use a result of Lang-Weil in [28], which asserts that for any algebraic variety $V$ defined over $\mathbb{Q}$ and any prime $p$, we have

$$
\# V\left(\mathbb{F}_{p}\right)=C_{V}(p) p^{\operatorname{dim} V}+O_{V}\left(p^{\operatorname{dim} V-1 / 2}\right)
$$

where $C_{V}(p)$ is the number of geometrically irreducible, top-dimensional components of $V$ which are defined over $\mathbb{F}_{p}$. We then have

$$
\rho_{F}(p)=\tau_{F}(p) p^{n-1}+O_{F}\left(p^{n-3 / 2}\right) .
$$

Multiplicativity of $\rho_{F}$ then yields

$$
\begin{aligned}
\rho_{F}(m) & =\prod_{p \mid m}\left(\tau_{F}(p) p^{n-1}+O_{F}\left(p^{n-3 / 2}\right)\right) \\
& =m^{n-1} \prod_{p \mid m}\left(\tau_{F}(p)+O_{F}\left(p^{-1 / 2}\right)\right) \\
& =O_{F}\left(m^{n-1} \tau_{F}(m) \sigma_{-1 / 4}(m)\right) .
\end{aligned}
$$

We remark that Lemma 2.1 implies that the infinite product

$$
\prod_{p}\left(1-\frac{\rho_{F}\left(p^{k}\right)}{p^{n k}}\right)
$$

converges. This is because

$$
\frac{\rho_{F}\left(p^{k}\right)}{p^{n k}}=O\left(\frac{1}{p^{k}}+\frac{1}{p^{n}}\right)=O\left(\frac{1}{p^{2}}\right)
$$

since $k, n \geq 2$ by assumption.

We give an estimate for $N_{1}(B)$. Define, for a positive integer $b$, the quantity

$$
N(b, B)=\#\left\{\mathbf{x} \in \mathbb{Z}^{n} \cap[-B, B]^{n}: b^{k} \mid F(\mathbf{x})\right\}
$$


Then from the familiar property of the Mobius function $\mu$, we have

$$
\begin{aligned}
N_{1}(B) & =\sum_{\substack{b \in \mathbb{N} \\
p \mid b \Rightarrow p \leq \xi_{1}}} \mu(b) N(b, B) \\
& =\sum_{\substack{b \in \mathbb{N} \\
p \mid b \Rightarrow p \leq \xi_{1}}} \mu(b) \rho_{F}\left(b^{k}\right)\left(\frac{B^{n}}{b^{n k}}+O\left(\frac{B^{n-1}}{b^{(n-1) k}}+1\right)\right) \\
& =B^{n} \prod_{p \leq \xi_{1}}\left(1-\frac{\rho_{F}\left(p^{k}\right)}{p^{n k}}\right)+O\left(\sum_{\substack{b \in \mathbb{N} \\
p \mid b \Rightarrow p \leq \xi_{1}}} \rho_{F}\left(b^{k}\right)\left(\frac{B^{n-1}}{b^{(n-1) k}}+1\right)\right) .
\end{aligned}
$$

By the theorem of Rosser and Schoenfeld [35], it follows that for all $\varepsilon>0$ and some $C^{\prime}>0$ we have

$$
\prod_{p \leq \xi_{1}} p \leq e^{2 \xi_{1}}=O\left((\log B)^{\frac{C^{\prime}}{\log _{3} B}}\right)=O_{\varepsilon}\left(B^{\varepsilon}\right)
$$

by (2.2). Hence, we obtain via Lemma 2.1 that, for any $\varepsilon>0$,

$$
N_{1}(B)=B^{n} \prod_{p \leq \xi_{1}}\left(1-\frac{\rho_{F}\left(p^{k}\right)}{p^{n k}}\right)+O\left(\sum_{b \ll_{\varepsilon} B^{\varepsilon}} B^{n-1+\varepsilon}+b^{n(k-1)+\varepsilon}+b^{k(n-1)+\varepsilon}\right) .
$$

We then see that

$$
N_{1}(B)=B^{n} \prod_{p \leq \xi_{1}}\left(1-\frac{\rho_{F}\left(p^{k}\right)}{p^{n k}}\right)+O_{\varepsilon}\left(B^{n-1+\varepsilon}\right) .
$$

As $B \rightarrow \infty$, the partial product in (2.9) tends to the convergent product in Theorem 1.1. thus it suffices to show that $N_{2}(B), N_{3}(B)$ are error terms.

In the next section we will see that we can obtain good estimates for $N_{2}(B)$ even when $\xi_{2}$ is as large as $B^{n}(\log B)^{2 / 3}$. Let

$$
F\left(x_{1}, \cdots, x_{n}\right)=\mathcal{F}_{1}(\mathbf{x}) \cdots \mathcal{F}_{r}(\mathbf{x}),
$$

where each $\mathcal{F}_{i}$ is irreducible over $\mathbb{Q}$ for $i=1, \cdots, r$. Here $d=\max _{1 \leq j \leq r} \operatorname{deg} \mathcal{F}_{j}$. Let us write

$$
\begin{gathered}
N_{2}^{(j)}(B)=\#\left\{\mathbf{x} \in \mathbb{Z}^{n}:\left|x_{i}\right| \leq B \text {, there exists } p \in\left(\xi_{1}, \xi_{2}\right] \text { s.t. } p^{k} \mid \mathcal{F}_{j}(\mathbf{x}),\right. \text { and } \\
\text { if } \left.p^{k} \mid \mathcal{F}_{j}(\mathbf{x}) \text {, then } p>\xi_{1}\right\},
\end{gathered}
$$

and

$$
\begin{gathered}
N_{3}^{(j)}(B)=\#\left\{\mathbf{x} \in \mathbb{Z}^{n}:\left|x_{i}\right| \leq B \text {, there exists } p>\xi_{2} \text { s.t. } p^{k} \mid \mathcal{F}_{j}(\mathbf{x}),\right. \\
\left.p^{2} \nmid F_{j}(\mathbf{x}) \text { for } \xi_{1}<p \leq \xi_{2} \text {, and if } p^{k} \mid \mathcal{F}_{j}(\mathbf{x}) \text {, then } p>\xi_{2}\right\} .
\end{gathered}
$$

If $\mathbf{x}$ is counted by $N_{2}(B)$ (respectively $N_{3}(B)$ ) but not by $N_{2}^{(j)}(B)$ (respectively $\left.N_{3}^{(j)}(B)\right)$ for $j=1, \cdots, r$, then there must exist $j_{1}<j_{2}$ and a positive integer $k^{\prime}<k$ such that

$$
\mathcal{F}_{j_{1}}(\mathbf{x}) \equiv 0 \quad\left(\bmod p^{k^{\prime}}\right) \text { and } \mathcal{F}_{j_{2}}(\mathbf{x}) \equiv 0 \quad\left(\bmod p^{k-k^{\prime}}\right)
$$


However, this can only happen if $p \mid \Delta(F)$, so this situation can be avoided if $B$ is chosen sufficiently large. Hence, we have

$$
N_{2}(B) \leq \sum_{j=1}^{r} N_{2}^{(j)}(B)
$$

and

$$
N_{3}(B) \leq \sum_{j=1}^{r} N_{3}^{(j)}(B) .
$$

It therefore suffices to deal with the case when $F$ is irreducible over $\mathbb{Q}$ and $d=\operatorname{deg} F$.

\section{Geometry of Numbers}

In this section we shall give an estimate for $N_{2}(B)$. To do so, we show that for each modulus $m$ we can reduce the problem to counting integer points of bounded height in a finite number $\mathcal{N}_{F}$ of lattices, the important feature being that $\mathcal{N}_{F}$ is dependent only on $F$.

Lemma 3.1. Let $F \in \mathbb{Z}\left[x_{1}, \cdots, x_{n}\right]$ be an incomplete norm form of degree $d>n$. Let $p \nmid \Delta(F)$ be a prime, and let $\mathbf{a}=\left(a_{1}, \cdots, a_{n}\right) \in \mathbb{Z}^{n}$ be a solution to the congruence

$$
F(\mathbf{x}) \equiv 0 \quad\left(\bmod p^{2}\right) .
$$

Then a lies on a finite number $\mathcal{N}_{F}$ of lattices $\Lambda \subset \mathbb{Z}^{n}$. Moreover, for each such lattice $\Lambda$, we have $\operatorname{det} \Lambda \geq p^{2}$.

Proof. By the same argument as that in Section 5 of [26], we can factor $F$ into

$$
F(\mathbf{x})=F^{*}(\mathbf{x}) \prod_{i=1}^{\tau_{F}(p)} \mathcal{L}_{i}(\mathbf{x})
$$

where $\mathcal{L}_{i}(\mathbf{x})=v_{1}^{(i)} x_{1}+v_{2}^{(i)} x_{2}+\cdots+v_{n}^{(i)} x_{n}$ are defined over $\mathbb{Z}_{p}$, while $F^{*}(\mathbf{x})$ is a form defined over $\mathbb{Z}_{p}$. Suppose that $\mathbf{a}=\left(a_{1}, \cdots, a_{n}\right) \in \mathbb{Z}^{n}$ is a solution to the congruence

$$
F(\mathbf{a}) \equiv 0 \quad\left(\bmod p^{2}\right) .
$$

Then $\mathbf{a}$ is of one of the following types:

(a) There exists exactly one $i, 1 \leq i \leq \tau_{F}(p)$ such that $\mathcal{L}_{i}(\mathbf{a}) \equiv 0\left(\bmod p^{2}\right)$, while $\mathcal{L}_{j}(\mathbf{a}) \not \equiv 0(\bmod p)$ for $j \neq i$, and $F^{*}(\mathbf{a}) \not \equiv 0(\bmod p)$.

(b) There exist $1 \leq i_{1}<i_{2} \leq \tau_{F}(p)$ such that

$$
\mathcal{L}_{i_{1}}(\mathbf{a}) \equiv \mathcal{L}_{i_{2}}(\mathbf{a}) \equiv 0 \quad(\bmod p) .
$$

(c) $F^{*}(\mathbf{a}) \equiv 0(\bmod p)$.

If $\mathbf{a}$ is of type (a), then a lies in the union of at most $\tau_{F}(p) \leq d$ lattices of determinant $p^{2}$. If $\mathbf{a}$ is of type (b), then there are two further sub-cases. Firstly, and more simply, there exist two indices $i_{1}<i_{2}$ and an integer $t$ such that

$$
\mathcal{L}_{i_{1}}(\mathbf{x}) \equiv t \mathcal{L}_{i_{2}}(\mathbf{x}) \quad(\bmod p) .
$$

If (3.1) holds, then it follows that $\Delta(F) \equiv 0(\bmod p)$, hence $p$ divides the discriminant $\Delta(F)$ of $F$. Thus, there are only finitely many primes for which this could happen. 
Otherwise, a lies on the intersection of two distinct lattices $\Lambda_{1}, \Lambda_{2}$ of determinant $p$, defined by

$$
\Lambda_{1}=\left\{\mathbf{x} \in \mathbb{Z}^{n}: \mathbf{x} \cdot \mathbf{a}_{1} \equiv 0 \quad(\bmod p)\right\}
$$

and

$$
\Lambda_{2}=\left\{\mathbf{x} \in \mathbb{Z}^{n}: \mathbf{x} \cdot \mathbf{a}_{2} \equiv 0 \quad(\bmod p)\right\},
$$

where $\mathbf{a}_{1}, \mathbf{a}_{2}$ are two non-proportional non-zero vectors modulo $p$. Now let $\phi_{1}, \phi_{2}$ be homomorphisms from $\mathbb{Z}^{n}$ to $\mathbb{F}_{p}$ defined by

$$
\phi_{1}(\mathbf{x})=\mathbf{a}_{1} \cdot \mathbf{x} \quad(\bmod p)
$$

and

$$
\phi_{2}(\mathbf{x})=\mathbf{a}_{2} \cdot \mathbf{x} \quad(\bmod p) .
$$

Then $\Lambda_{1}, \Lambda_{2}$ are the kernels of $\phi_{1}, \phi_{2}$ respectively. Now let $\phi$ be defined by $\phi: \mathbb{Z}^{n} \rightarrow$ $(\mathbb{Z} / p \mathbb{Z})^{2}, \phi(\mathbf{x})=\left(\phi_{1}(\mathbf{x}), \phi_{2}(\mathbf{x})\right)$. The image of $\phi$ is the full set $(\mathbb{Z} / p \mathbb{Z})^{2}$ whenever $\mathbf{a}_{1}, \mathbf{a}_{2}$ are not proportional modulo $p$. Hence, a lies in a lattice of determinant at least $p^{2}$. Further, there are at most $\tau_{F}(p)^{2} \leq d^{2}$ such lattices.

If $\mathbf{a}$ is of type (c), then modulo $p$ there exists a linear factor $\mathcal{L}_{j}$ of $F^{*}$ which is not defined over $\mathbb{F}_{p}$ such that $\mathcal{L}_{j}(\mathbf{a}) \equiv 0(\bmod p)$. Let $s$ be the degree of the field of definition of $\mathcal{L}_{j}$ over $\mathbb{F}_{p}$. By assumption, we have $s \geq 2$. Then $\mathcal{L}_{j}$ can be written as

$$
\mathcal{L}_{j}=\alpha_{1} \mathcal{L}_{j, 1}+\cdots+\alpha_{s} \mathcal{L}_{j, s},
$$

where $\mathcal{L}_{j, i}$ are linear forms with coefficients in $\mathbb{F}_{p}$ and $\alpha_{1}, \cdots, \alpha_{s}$ is a basis of $\mathbb{F}_{p^{s}}$ over $\mathbb{F}_{p}$. In particular, $\alpha_{1}, \cdots, \alpha_{s}$ are linearly independent over $\mathbb{F}_{p}$. Therefore, $\mathcal{L}_{j}(\mathbf{a}) \equiv 0$ $(\bmod p)$ implies that $\mathcal{L}_{j, i}(\mathbf{a}) \equiv 0(\bmod p)$ for $i=1, \cdots, s$. It thus follows that a lies in the intersection of the lattice in $\mathbb{Z}^{n}$ given by the linear forms $\mathcal{L}_{j, 1}, \mathcal{L}_{j, 2}$, hence by the same argument it follows that a lies in a lattice of determinant at least $p^{2}$. Moreover, the number of such lattices is at most $d^{2}$.

Now we generalize Lemma 1 in [15] (see also [18]) for norm forms in $n \geq 2$ variables. Indeed, we will prove the following:

Lemma 3.2. Let $\Lambda \subset \mathbb{Z}^{n}$ be a lattice of determinant $m$. For $\mathbf{x} \in \mathbb{Z}^{n}$ denote by $H(\mathbf{x})$ the sup norm of $\mathbf{x}$. Put

$$
N_{\Lambda}(B)=\left\{\mathbf{x} \in \mathbb{Z}^{n}: H(\mathbf{x}) \leq B\right\}
$$

and put $M_{\Lambda}$ for the sup norm of the shortest vector in $\Lambda$. Then

$$
N_{\Lambda}(B) \ll_{n} \frac{B^{n}}{m}+O\left(\frac{B^{n-1}}{M_{\Lambda}^{n-1}}+1\right) .
$$

Proof. Let $\mathbf{x}_{1}=\left(x_{1}^{(1)}, \cdots, x_{n}^{(1)}\right)$ be one of the shortest vectors with respect to sup norm. Without loss of generality, we may assume that $\left|x_{1}^{(0)}\right|=M_{\Lambda}$. Observe that $M_{\Lambda} \leq m^{1 / n}$. To see this, let $l=l(m)$ denote the smallest positive integer such that $(l+1)^{n}>m$. Then there exist two distinct vectors $\mathbf{a}_{1}, \mathbf{a}_{2}$ such that the coordinates of both vectors are at most $l / 2$ in absolute value and

$$
\mathbf{a}_{1} \equiv \mathbf{a}_{2} \quad(\bmod m),
$$


whence their difference $\mathbf{a}_{1}-\mathbf{a}_{2}$ lies in $\mathcal{L}$ and $H\left(\mathbf{a}_{1}-\mathbf{a}_{2}\right) \leq m^{1 / n}$.

By Lemma 4.3 in [4], there exist vectors $\mathbf{x}_{2}, \cdots, \mathbf{x}_{n} \in \mathcal{L}$ such that

$$
m \leq \prod_{j=1}^{n} H\left(\mathbf{x}_{j}\right) \ll_{n} m
$$

and for all vectors $\mathbf{x} \in \mathcal{L}$, if we write

$$
\mathbf{x}=\sum_{j=1}^{n} \lambda_{j} \mathbf{x}_{j}
$$

we have

$$
\left|\lambda_{j}\right| \ll_{n} \frac{H(\mathbf{x})}{H\left(\mathbf{x}_{j}\right)} .
$$

In particular, for a vector $\mathbf{x}$ counted by $N_{\Lambda}(B)$, we have

$$
\left|\lambda_{j}\right| \ll_{n} \frac{B}{H\left(\mathbf{x}_{j}\right)} .
$$

By observing that $H\left(\mathbf{x}_{j}\right) \geq M_{\Lambda}$ for $j=1, \cdots, n$, we obtain the bound

$$
\begin{aligned}
N_{\Lambda}(B) & \ll_{n} \prod_{j=1}^{n}\left(1+\frac{B}{H\left(\mathbf{x}_{j}\right)}\right) \\
& \ll_{n} \frac{B^{n}}{m}+\frac{B^{n-1}}{M_{\Lambda}^{n-1}}+\cdots+1 .
\end{aligned}
$$

Hence we obtain the consequence of the lemma.

For each prime $p$, we denote by $\mathcal{U}_{p}$ the set of lattices containing the solutions to the congruence $F(\mathbf{x}) \equiv 0\left(\bmod p^{2}\right)$. For each $\Lambda \in \mathcal{U}_{p}$, we say that $\Lambda$ is of type a), b), or c) if $\Lambda$ arises from a solution a to $F(\mathbf{x}) \equiv 0\left(\bmod p^{2}\right)$ of type a), b), or c) in the proof of Lemma 3.1. Then write $F_{\Lambda}$ to be equal to:

(a) $L_{i}(\mathbf{x})$, if $\Lambda$ is of type a) and $\mathcal{L}_{i}$ is the unique linear form associated to $\Lambda$;

(b) $\mathcal{L}_{i_{1}} \cdots \mathcal{L}_{i_{s}}$, where $\mathcal{L}_{i_{1}}, \cdots, \mathcal{L}_{i_{s}}$ are the linear factors of $F$ defined over $\mathbb{F}_{p}$ which vanish on $\Lambda$ modulo $p$ when $\Lambda$ is of type b); and

(c) $F^{*}$ if $\Lambda$ is of type c).

We now estimate $N_{2}(B)$ via the following lemma:

Lemma 3.3. The error term $N_{2}(B)$ satisfies

$$
N_{2}(B)=O_{n}\left(B^{n}\left(\xi_{1}^{-1}+(\log B)^{-1 / 3 n}\right)\right) .
$$

Proof. Let $\mathcal{U}_{p}$ denote the set of at most $\mathcal{N}_{F}$ many lattices $\Lambda$, each with determinant at least $p^{2}$ by Lemma 3.1, which contains all of the solutions to $F(\mathbf{x}) \equiv 0\left(\bmod p^{2}\right)$. Then

$$
N_{2}(B) \ll_{n} \sum_{\xi_{1}<p \leq B^{n}(\log B)^{2 / 3}} \sum_{\Lambda \in \mathcal{U}_{p}} N_{\Lambda}(B) .
$$


By Lemma 3.2, it follows that

$$
N_{2}(B) \ll_{n} \sum_{\xi_{1}<p \leq B^{n}(\log B)^{2 / 3}} \sum_{\Lambda \in \mathcal{U}_{p}}\left(\frac{B^{n}}{p^{2}}+\frac{B^{n-1}}{M_{\Lambda}^{n-1}}+1\right) .
$$

We first consider consider the term

$$
\sum_{\xi_{1}<p \leq \xi_{2}} \sum_{\Lambda \in \mathcal{U}_{p}} \frac{B^{n}}{p^{2}}
$$

The sum

$$
\sum_{p>\xi_{1}} \sum_{1 \leq j \leq \mathcal{N}_{F}} \frac{1}{p^{2}}
$$

converges and is bounded by $O_{F}\left(\xi_{1}^{-1}\right)$. Now we look at the sum

$$
\sum_{\xi_{1}<p \leq \xi_{2}} \sum_{\Lambda \in \mathcal{U}_{p}} \frac{B^{n-1}}{M_{\Lambda}^{n-1}}
$$

We break the above sum into three sub-sums $S_{1}, S_{2}$, and $S_{3} . S_{1}$ will consist of the contribution from those primes $\xi_{1}<p \leq B$. In this case, we have

$$
\begin{aligned}
S_{1} & =\sum_{\xi_{1}<p \leq B} \sum_{\Lambda \in \mathcal{U}_{p}} \frac{B^{n-1}}{M_{\Lambda}^{n-1}} \\
& \ll B^{n-1} \sum_{1 \leq j \leq \mathcal{N}_{F}} \sum_{p \leq B} 1 \\
& \ll \frac{B^{n}}{\log B}
\end{aligned}
$$

where we used the trivial estimate that $M_{\Lambda} \geq 1$.

$S_{2}$ will be the sub-sum consisting of those $M_{\Lambda} \geq B(\log B)^{-1 / 3 n}$. In this case, we have

$$
\begin{aligned}
S_{2} & \ll_{d} \sum_{\xi_{1}<p \leq B^{n}(\log B)^{2 / 3}} \sum_{\Lambda \in \mathcal{U}_{p}} \frac{B^{n-1}(\log B)^{(n-1) / 3 n}}{B^{n-1}} \\
& \ll_{d}(\log B)^{\frac{(n-1)}{3 n}} \frac{B^{n}(\log B)^{2 / 3}}{\log B} \\
& \ll_{d} B^{n}(\log B)^{-1 / 3 n} .
\end{aligned}
$$


Finally, $S_{3}$ will denote the sub-sum consisting of those primes $p>B$ and $M_{\Lambda} \leq$ $B(\log B)^{-1 / 3 n}$. We then have

$$
\begin{aligned}
S_{3} & \ll \sum_{0<\left|x_{1}^{(1)}\right|, \cdots,\left|x_{n}^{(1)}\right| \leq B(\log B)^{-1 / 3 n}} \sum_{\substack{M_{\Lambda} \in \mathcal{U}_{p} \\
p^{2} \mid F_{\Lambda}\left(\mathbf{x}_{1}\right) \\
p>B}} \frac{B^{n-1}}{M_{\Lambda}^{n-1}} \\
& \ll B^{n-1} \sum_{0<\left|x_{1}^{(1)}\right| \leq B(\log B)^{-1 / 3 n}} \frac{1}{\left|x_{1}^{(1)}\right|^{n-1}} \sum_{\substack{0 \leq\left|x_{2}^{(1)}\right|, \cdots,\left|x_{n}^{(1)}\right| \leq\left|x_{1}^{(1)}\right| p^{2} \mid F\left(\mathbf{x}_{1}\right) \\
p>B}} 1 \\
& \ll B^{n-1} B(\log B)^{-1 / 3 n},
\end{aligned}
$$

the last inequality following form the fact that at most $\lfloor d / 2\rfloor+1$ many primes with $p>B$ can satisfy $p^{2} \mid F\left(\mathbf{x}_{1}\right)$, since $\left\|\mathbf{x}_{1}\right\| \leq B$.

Finally, the last term needing to be estimated is

$$
\sum_{\xi_{1}<p \leq B^{n}(\log B)^{2 / 3}} \sum_{\Lambda \in \mathcal{U}_{p}} 1 .
$$

This is bounded by the number of primes in the interval $\left[\xi_{1}, B^{n}(\log B)^{2 / 3}\right]$, which by the prime number theorem is $O\left(B^{n}(\log B)^{2 / 3} / \log B\right)=O\left(B^{n}(\log B)^{-1 / 3}\right)$, and so constitutes a negligible error term.

\section{The Ekedahl Sieve}

In this section, we use the following result of Ekedahl in [7] to handle certain contributions to $N_{3}(B)$. The version below was formulated by Bhargava and Shankar in [2]:

Proposition 4.1 (Ekedahl sieve). Let $\mathcal{B}$ be a compact region in $\mathbb{R}^{n}$ having finite measure, and let $Y$ be any closed subscheme of $\mathbb{A}_{\mathbb{Z}}^{n}$ of co-dimension $s \geq 2$. Let $r$ and $M$ be positive real numbers. Then we have

$$
\begin{gathered}
\#\left\{\mathbf{x} \in r \mathcal{B} \cap \mathbb{Z}^{n}: \mathbf{x} \quad(\bmod p) \in Y\left(\mathbb{F}_{p}\right) \text { for some prime } p>M\right\} \\
=O\left(\frac{r^{n}}{M^{s-1} \log M}+r^{n-s+1}\right) .
\end{gathered}
$$

We factor $F$ into linear factors over $\overline{\mathbb{Q}}$, where

$$
F(\mathbf{x})=\prod_{j=1}^{d}\left(\psi_{1}^{(j)} x_{1}+\cdots+\psi_{n}^{(j)} x_{n}\right)=\prod_{i=1}^{d} L_{i}(\mathbf{x}) .
$$

Let $Y_{i, j}$ denote the variety defined by $L_{i}(\mathbf{x})=L_{j}(\mathbf{x})=0$, and let $Y=\bigcup_{1 \leq i<j \leq n} Y_{i, j}$. Since $Y$ is invariant under the action of $\operatorname{Gal}(\overline{\mathbb{Q}} / \mathbb{Q})$, it is defined over $\mathbb{Q}$. Moreover it has co-dimension at least two in $\mathbb{A}_{\mathbb{Z}}^{n}$. Let $p$ be a prime. Over $\mathbb{Z}_{p}$, we have the factorization (see [25]) of $F$ into

$$
F(\mathbf{x})=F^{*}(\mathbf{x}) \prod_{i=1}^{\tau_{F}(p)} \mathcal{L}_{i}(\mathbf{x})
$$


where $F^{*}, \mathcal{L}_{i}$ have $\mathbb{Z}_{p}$-coefficients and $F^{*}$ does not have linear factors over $\mathbb{Q}_{p}$. Let $\mathcal{S}_{p}$ be those congruence classes $\mathbf{x}$ in $(\mathbb{Z} / p \mathbb{Z})^{n}=\mathbb{F}_{p}^{n}$ such that either

(a) There exist $1 \leq i<j \leq \tau_{F}(p)$ such that $\mathcal{L}_{i}(\mathbf{x}) \equiv \mathcal{L}_{j}(\mathbf{x}) \equiv 0(\bmod p)$, or

(b) $F^{*}(\mathbf{x}) \equiv 0(\bmod p)$.

Since linear factors of $F^{*}$ are not defined over $\mathbb{F}_{p}$ and hence has a non-trivial conjugate, it follows that whenever $\mathbf{a} \in \mathcal{S}_{p}$ that $\mathbf{a} \in Y\left(\mathbb{F}_{p}\right)$. We then have the following consequence of Ekedahl's sieve:

Lemma 4.2. Let $N_{3}^{*}(B)$ denote the number of elements $\mathbf{x} \in \mathbb{Z}^{n} \cap[-B, B]^{n}$ for which $\mathbf{x}(\bmod p) \in \mathcal{S}_{p}$ for some $p>\xi_{1}$. Then

$$
N_{3}^{*}(B)=O\left(\frac{B^{n}}{\xi_{1} \log \xi_{1}}+B^{n-1}\right) .
$$

Note that Lemma 4.2 completes the proof of Lemma 3.3.

\section{The SElBerg SIEVE}

In this section we use a variant of the Selberg sieve to give an upper bound for $N_{3}(B)$. Our main goal in this section is to establish the following proposition:

Proposition 5.1. Let $N_{3}(B)$ be as given in (2.6). Then $N_{3}(B)=o\left(B^{n}\right)$.

Proposition 5.1 will follow from Lemmas 5.4, 5.6, 5.7, 5.10, and 5.12 below as well as Lemma 4.2, Consider the set

$$
\mathcal{N}_{3}^{\dagger}(B)=\left\{\begin{array}{l}
\mathbf{x} \in \mathbb{Z}^{n} \cap[-B, B]^{n} \mid \begin{array}{c}
F(\mathbf{x})=u q^{k}, u \text { is indivisible by } p^{k} \text { for } p \leq \xi_{1}, \\
\text { indivisible by } p^{2} \text { for } \xi_{1}<p \leq \xi_{2}, \\
q \text { is a prime exceeding } \xi_{2}, \mathbf{x} \notin \mathcal{S}_{p} \text { for all } p \mid u .
\end{array}
\end{array}\right\},
$$

and put $N_{3}^{\dagger}(B)=\# \mathcal{N}_{3}^{\dagger}(B)$. Observe that

$$
N_{3}(B)=N_{3}^{\dagger}(B)+N_{3}^{*}(B) \text {. }
$$

We shall establish the following preliminary result:

Lemma 5.2. Let $\mathbf{x} \in \mathcal{N}_{3}^{\dagger}(B)$ and $u, q$ be as in (5.1). Then we have

$$
u=O\left(B^{2}(\log B)^{-2 k / 3}\right) \text {. }
$$

Furthermore, $u$ can be written as $u=u_{1} u_{2}$, where $u_{1}$ divides

$$
C\left(\xi_{1}\right)=\prod_{p \leq \xi_{1}} p^{k-1}
$$

and $u_{2}$ is square-free with each prime divisor $p$ of $u_{2}$ satisfying $\xi_{1}<p \leq \xi_{2}$.

Proof. Observe that from $F(\mathbf{x})=u q^{k}$ and our assumptions on $q$, we have

$$
u=O\left(B^{d} \xi_{2}^{-k}\right) \text {. }
$$

By (1.6) and (2.3), there exists an absolute positive constant $C_{1}$ such that

$$
\begin{aligned}
|u| & <C_{1} B^{d-k n}(\log B)^{-2 k / 3} \\
& \leq C_{1} B^{d-d+2}(\log B)^{-2 k / 3} \\
& =C_{1} B^{2}(\log B)^{-2 k / 3} .
\end{aligned}
$$


We now factor $u$ into two factors $u_{1}$ and $u_{2}$, where $u_{1}$ consists of only prime factors less than $\xi_{1}$. We observe that since we have accounted for small prime powers via our treatment of $N_{1}(B)$, we have that $u_{1}$ divides $\prod_{p \leq \xi_{1}} p^{k-1}$. The factor $u_{2}$, then, will be composed of prime factors larger than $\xi_{1}$. Further, it must be square-free. This is because, by definition, the prime factors of $u$ between $\xi_{1}$ and $\xi_{2}$ divide $u$ exactly once, and $u$ cannot have a prime factor exceeding $\xi_{2}$, since otherwise

$$
u q^{k} \gg B^{n(k+1)} \log B \gg B^{d} \log B,
$$

which contradicts $\mathbf{x} \in[-B, B]^{n}$ for $B$ sufficiently large.

For each square-free integer $u_{2}$ such that each prime divisor $p$ of $u_{2}$ satisfies $\xi_{1}<$ $p \leq \xi_{2}$, put

$$
\mathcal{D}\left(u_{2}\right)=\prod_{\substack{\xi_{1}<p \leq \frac{1}{12} \log \left(B^{2} u_{2}^{-1}\right) \\ p \nmid u_{2} \\ p \equiv 1}} p .
$$

We then have the following lemma:

Lemma 5.3. Let $u_{2}$ be a square-free integer such that all of its prime divisors are between $\xi_{1}$ and $\xi_{2}$. Let $\omega(m)$ denote the number of distinct prime divisors of $m$. Let $\mathcal{D}\left(u_{2}\right)$ be as in (5.3). If $q>\xi_{2}$ is a prime, then there exists exactly $k^{\omega(\mathcal{D})}$ residue classes $\left\{\mathfrak{d}_{1}, \cdots, \mathfrak{d}_{k^{\omega(D)}}\right\}$ such that

$$
\mathfrak{d}_{j}^{k} \equiv q^{k} \quad(\bmod \mathcal{D})
$$

for $j=1, \cdots, k^{\omega(D)}$.

Proof. Since all prime divisors of $\mathcal{D}$ are $O(\log B)$, it follows that $q^{k}$ is a proper $k$-th power residue modulo $\mathcal{D}$. Now consider the family of all $k$-th power residues modulo $\mathcal{D}$. By our choice of $\mathcal{D}$, we have that $k \mid \varphi(\mathcal{D})$, so that the family of $k$-th power residues is not the set of all residues modulo $\mathcal{D}$. For each $p \mid \mathcal{D}, q^{k}$ has $k$ pre-images modulo $p$, meaning there exist $k$ distinct elements $\mathfrak{q}_{1}, \cdots, \mathfrak{q}_{k}$ in $\{0,1, \cdots, p-1\}$ such that $\mathfrak{q}_{j}^{k} \equiv q^{k}(\bmod \mathcal{Q})$. For a positive integer $l$ let us write $\omega(l)$ for the number of distinct prime divisors of $l$. Then it follows from the Chinese Remainder Theorem that there exist $k^{\omega(\mathcal{D})}$ residue classes $\left\{\mathfrak{d}_{1}, \cdots, \mathfrak{d}_{k^{\omega(\mathcal{D})}}\right\}$ modulo $\mathcal{D}$ such that $\mathfrak{d}_{j}^{k} \equiv q^{k}(\bmod \mathcal{D})$.

Let $C_{1}$ be as in Lemma 5.2 , and put $\xi_{3}=C_{1} B^{2}(\log B)^{-2 k / 3}$. Lemmas 5.2 and 5.3 have the following consequence, which is crucial for our estimation of $N_{3}(B)$ :

Lemma 5.4. Let $u_{1}$ be a divisor of $C\left(\xi_{1}\right)$ and let $u_{2}$ a square-free integer whose prime divisors $p$ satisfy $\xi_{1}<p \leq \xi_{3}$. Let $H_{u_{1}, u_{2}}(B)$ be the number of solutions $\left(m_{1}, \cdots, m_{n}\right) \in \mathbb{Z}^{n} \cap[-B, B]^{n}$ to the following three congruences:

$$
\begin{aligned}
& F\left(m_{1}, \cdots, m_{n}\right) \equiv 0 \quad\left(\bmod u_{1}\right), \\
& F\left(m_{1}, \cdots, m_{n}\right) \equiv 0 \quad\left(\bmod u_{2}\right),
\end{aligned}
$$

and for $0 \leq s<\mathcal{D}$, the solutions to the congruences

$$
F\left(m_{1}, \cdots, m_{n}\right) \equiv u_{1} u_{2} s^{k} \quad(\bmod \mathcal{D})
$$


such that $\left(m_{1}, \cdots, m_{n}\right)(\bmod p) \notin \mathcal{S}_{p}$ for $p \mid u_{1} u_{2}$. Then we have

$$
N_{3}(B) \leq \sum_{\substack{u_{1} \mid C\left(\xi_{1}\right) \\ u_{2} \leq \xi_{3}}} \frac{H_{u_{1}, u_{2}}(B)}{k^{\omega(\mathcal{D})}}+N_{3}^{*}(B)
$$

Proof. (5.7) follows from the fact that the solutions to (5.6) can be partitioned into sets of cardinality $k^{\omega(\mathcal{D})}$ by Lemma 5.3 .

In view of Lemma 4.2, we shall be primarily concerned with the term

$$
N_{3}^{\dagger}(B)=\sum_{\substack{u_{1} \mid C\left(\xi_{1}\right) \\ u_{2} \leq \xi_{3}}} \frac{H_{u_{1}, u_{2}}(B)}{k^{\omega(\mathcal{D})}}
$$

5.1. Selberg sieve weights. We now introduce the relevant Selberg sieve weights. Selberg devised an ingenious method to establish an upper bound for counting integer points in a box. To state this precisely, suppose that we wanted to count the set of points inside the box $[-B, B]^{n}$ satisfying a set of congruence conditions $\mathcal{R}_{l}$ modulo a positive integer $l$. Selberg introduced smooth functions $\gamma$ which satisfy the inequality

$$
\sum_{\substack{\left(m_{1}, \cdots, m_{n}\right) \in \mathbb{Z}^{n} \cap[-B, B]^{n} \\\left(m_{1}, \cdots, m_{n}\right) \in \mathcal{R}_{l}}} 1 \leq \sum_{\substack{\left(m_{1}, \cdots, m_{n}\right) \in \mathbb{Z}^{n} \\\left(m_{1}, \cdots, m_{n}\right) \in \mathcal{R}_{l}}} \gamma\left(m_{1}\right) \cdots \gamma\left(m_{n}\right),
$$

where $\gamma$ is an upper bound for the characteristic function $\chi_{B}(z)$ of the interval $[-B, B]$, tends to zero rapidly outside of this interval, and is sufficiently smooth to be conducive to Fourier analysis and the Poisson summation formula. This reduces various counting problems into a question about exponential sums, from which one can draw results from a vast literature, including the seminal works of Weil and Deligne.

Our choice of $\gamma$ is identical to that of Hooley's in [25]. Namely, we start with the function, first given by Beurling and later utilized by Selberg to establish the optimal general bound for the large sieve inequality:

$$
\operatorname{Beu}(z)=\left(\frac{\sin \pi z}{\pi}\right)^{2}\left(\sum_{n=0} \frac{1}{(z-n)^{2}}-\sum_{n=-\infty}^{-1} \frac{1}{(z-n)^{2}}+\frac{1}{2 z}\right) .
$$

For the interval $[-U, U]$ we construct the function

$$
g_{U}(z)=\frac{1}{2}(\operatorname{Beu}(U-z)+\operatorname{Beu}(U+z))
$$

which has the property that it is non-negative and majorizes the characteristic function of $[-U, U]$ (see [40]). Further, it satisfies the important property that its Fourier transform $\hat{g}_{U}(t)$ satisfies

$$
\hat{g}_{U}(t)= \begin{cases}2 U+1 & \text { if } t=0 \\ 0 & \text { if }|t|>U\end{cases}
$$

and

$$
\left|\hat{g}_{U}(t)\right| \leq 2 U+1
$$


We now define the function $\gamma$ as

$$
\gamma(z)=g_{1}\left(\frac{z}{B}\right)
$$

whence it follows that

$$
\hat{\gamma}(t)=B \hat{g}_{1}(B t) \text {. }
$$

It is clear that $\gamma(z) \geq \chi_{B}(z)$ for all real numbers $z$. Because of the smoothness of $\gamma$, we can evaluate the sum

$$
\sum_{\substack{\left(m_{1}, \cdots, m_{n}\right) \in \mathbb{Z}^{n} \\\left(m_{1}, \cdots, m_{n}\right) \in \mathcal{R}_{l}}} \gamma\left(m_{1}\right) \cdots \gamma\left(m_{n}\right)
$$

via Poisson summation. We have the following lemma, which is standard:

Lemma 5.5. Let $l$ be a positive integer, and let $\mathcal{R}_{l}$ be a subset of $(\mathbb{Z} / l \mathbb{Z})^{n}$. Let $\gamma$ be as in (5.11), and put

$$
M_{\mathcal{R}_{l}}(B)=\sum_{\substack{\left(m_{1}, \cdots, m_{n}\right) \in \mathbb{Z}^{n} \\\left(m_{1}, \cdots, m_{n}\right) \in \mathcal{R}_{l}}} \gamma\left(m_{1}\right) \cdots \gamma\left(m_{n}\right)
$$

Let

$$
E_{\mathcal{R}_{l}}\left(t_{1}, \cdots, t_{n} ; l\right)=\sum_{\left(a_{1}, \cdots, a_{n}\right) \in \mathcal{R}_{l}} e^{-2 \pi i\left(a_{1} t_{1}+\cdots+a_{n} t_{n}\right) / l} .
$$

Then

$$
M_{\mathcal{R}_{l}}(B)=\frac{1}{l^{n}} \sum_{\left(t_{1}, \cdots, t_{n}\right) \in \mathbb{Z}^{n}} \hat{\gamma}\left(\frac{t_{1}}{l}\right) \cdots \hat{\gamma}\left(\frac{t_{n}}{l}\right) E_{\mathcal{R}_{l}}\left(t_{1}, \cdots, t_{n} ; l\right) .
$$

Proof. See [26].

We shall decompose $M_{\mathcal{R}_{l}}(B)$ into two terms, given by

$$
M_{\mathcal{R}_{l}}(B)=M_{\mathcal{R}_{l}}^{+}(B)+O\left(M_{\mathcal{R}_{l}}^{++}(B)\right)
$$

where

and

$$
M_{\mathcal{R}_{l}}^{+}(B)=\frac{1}{l^{n}}(\hat{\gamma}(0))^{n} E_{\mathcal{R}_{l}}(0, \cdots, 0 ; l)=\frac{(3 B)^{n} \# \mathcal{R}_{l}}{l^{n}}
$$

$$
M_{\mathcal{R}_{l}}^{++}(B)=\frac{B^{n}}{l^{n}} \sum_{\left|t_{i}\right| \leq l / B}^{\prime}\left|E_{\mathcal{R}_{l}}\left(t_{1}, \cdots, t_{n} ; l\right)\right|,
$$

where the symbol $\sum^{\prime}$ denotes that the tuple $(0, \cdots, 0)$ had been omitted. We then have the following:

Lemma 5.6. Let $l=u_{1} u_{2} \mathcal{D}$, where $u_{1}, u_{2}, \mathcal{D}$ are as in Lemma 5.4. Put $l=u_{1} u_{2} \mathcal{D}$, and let $\mathcal{R}_{l}=\mathcal{R}_{u_{1} u_{2} \mathcal{D}}$ denote the set of congruence classes modulo l satisfying (5.4), (5.5), and (5.6). Then

$$
N_{3}^{\dagger}(B) \leq \sum_{\substack{u_{1} \mid C\left(\xi_{1}\right) \\ u_{2} \leq \xi_{3}}} \frac{M_{\mathcal{R}_{l}}^{+}(B)}{k^{\omega(\mathcal{D})}}+O\left(\sum_{\substack{u_{1} \mid C\left(\xi_{1}\right) \\ u_{2} \leq \xi_{3}}} \frac{M_{\mathcal{R}_{l}}^{++}(B)}{k^{\omega(\mathcal{D})}}\right)
$$


Proof. This follows from (5.7), (5.8) and (5.14).

We put

$$
N_{4}(B)=\sum_{\substack{u_{1} \mid C\left(\xi_{1}\right) \\ u_{2} \leq \xi_{3}}} \frac{M_{\mathcal{R}_{l}}^{+}(B)}{k^{\omega(\mathcal{D})}} .
$$

Our next lemma gives us an estimate for $N_{4}(B)$ :

Lemma 5.7. Let $u_{1}, u_{2}, \mathcal{D}, l, \mathcal{R}_{l}$ be as in Lemma 5.6 and $N_{4}(B)$ as in (5.15). Then there exists a positive number $C_{4}$ such that

$$
N_{4}(B)=O\left(\frac{B^{n} \exp \left(2(n+1)(k-1) \xi_{1}\right)}{(\log B)^{C_{4} / \log _{3} B}}\right) .
$$

Proof. Let $\mathcal{R}_{u_{1}}, \mathcal{R}_{u_{2}}, \mathcal{R}_{\mathcal{D}}$ denote respectively the congruence classes corresponding to (5.4), (5.5) and (5.6), respectively. By the Chinese Remainder Theorem it follows that

$$
\# \mathcal{R}_{l}=\# \mathcal{R}_{u_{1}} \# \mathcal{R}_{u_{2}} \# \mathcal{R}_{\mathcal{D}}
$$

Since $u_{1} \mid C\left(\xi_{1}\right)$, it follows that $u_{1} \leq C\left(\xi_{1}\right)$. From its definition and the result of Rosser and Schoenfeld [35], we see that

$$
C\left(\xi_{1}\right) \leq \exp \left(2(k-1) \xi_{1}\right)
$$

For $\mathcal{R}_{u_{1}}$, we use the trivial bound $\# \mathcal{R}_{u_{1}}=O\left(u_{1}^{n}\right)=O\left(\exp \left(2 n(k-1) \xi_{1}\right)\right)$. We have $\# \mathcal{R}_{u_{2}}=O\left(u_{2}^{n-1} \tau_{F}\left(u_{2}\right) \sigma_{-1 / 4}\left(u_{2}\right)\right)$ by Lemma 2.1, since $u_{2}$ is square-free. Observe that $\operatorname{gcd}\left(u_{1} u_{2}, \mathcal{D}\right)=1$. By the theorem of Lang and Weil [28], which states that the number of points over $\mathbb{F}_{p}$, for a prime $p \mid \mathcal{D}$, on the variety defined by the congruence

$$
F\left(x_{1}, \cdots, x_{n}\right)-u_{1} u_{2} q^{k} \equiv 0 \quad(\bmod p),
$$

is

$$
p^{n}+O\left(p^{n-1 / 2}\right) .
$$

Then

$$
\# \mathcal{R}_{\mathcal{D}}=\prod_{p \mid \mathcal{D}}\left(p^{n}+O\left(p^{n-1 / 2}\right)\right)
$$

whence

$$
\# \mathcal{R}_{\mathcal{D}}=\mathcal{D}^{n} \prod_{p \mid \mathcal{D}}\left(1+O\left(p^{-1 / 2}\right)\right)=O\left(\mathcal{D}^{n} \sigma_{-1 / 4}(\mathcal{D})\right) .
$$

Thus, by (5.14), (5.16), and Lemma 2.1 we see that

$$
\begin{aligned}
N_{4}(B) & =O\left(\exp \left(2 n(k-1) \xi_{1}\right) \sum_{\substack{u_{1} \mid C\left(\xi_{1}\right) \\
u_{2} \leq \xi_{3}}} \frac{(3 B)^{n} u_{2}^{n-1} \tau_{F}\left(u_{2}\right) \sigma_{-1 / 4}\left(u_{2}\right) \mathcal{D}^{n} \sigma_{-1 / 4}(\mathcal{D})}{\left(u_{2} \mathcal{D}\right)^{n} k^{\omega(\mathcal{D})}}\right) \\
& =O\left(\exp \left(2 n(k-1) \xi_{1}\right) \sum_{\substack{u_{1} \mid C\left(\xi_{1}\right) \\
u_{2} \leq \xi_{3}}} \frac{B^{n} \sigma_{-1 / 4}\left(u_{2}\right) \tau_{F}\left(u_{2}\right) \sigma_{-1 / 4}(\mathcal{D})}{u_{2} k^{\omega(D)}}\right) \cdot
\end{aligned}
$$


Observe that

$$
\begin{aligned}
\sigma_{-1 / 4}(\mathcal{D}) & =\prod_{p \mid \mathcal{D}}\left(1+p^{-1 / 4}\right) \\
& =O\left(\left(\frac{2 k}{3}\right)^{\omega(\mathcal{D})}\right) .
\end{aligned}
$$

It follows that

$$
N_{4}(B)=O\left(\left(\exp \left(2 n(k-1) \xi_{1}\right) \sum_{\substack{u_{1} \mid C\left(\xi_{1}\right) \\ u_{2} \leq \xi_{3}}} \frac{B^{n} \tau_{F}\left(u_{2}\right) \sigma_{-1 / 4}\left(u_{2}\right)}{u_{2}(3 / 2)^{\omega(D)}}\right) .\right.
$$

Let us write

$$
\xi_{4}=\xi_{4}\left(u_{2}\right)=\frac{1}{12} \log \left(B^{2} u_{2}^{-1}\right)
$$

and

$$
\mathcal{D}^{\prime}=\mathcal{D}^{\prime}\left(u_{2}\right)=\prod_{p \leq \xi_{4}} p .
$$

Observe that as $B^{2} \xi_{3}^{-1} \rightarrow \infty$ as $B$ tends to infinity and $u_{2}^{-1} \gg \xi_{3}^{-1}$, we have

$$
\log \mathcal{D}^{\prime}=\sum_{p \leq \xi_{4}} \log p<\frac{12}{11} \xi_{4}
$$

for $B$ sufficiently large, say by Rosser and Schoenfeld [35]. From (5.3), we see that

$$
\mathcal{D} \leq \mathcal{D}^{\prime}<\exp \left(12 \xi_{4} / 11\right)=\left(\frac{B^{2}}{u_{2}}\right)^{1 / 11} .
$$

Next, we have

$$
\omega\left(\mathcal{D}^{\prime}\right)=\pi\left(\xi_{4} ; k, 1\right) \sim \frac{\xi_{4}}{\varphi(k) \log \xi_{4}},
$$

where $\pi(B ; q, a)$ is the counting function of primes $p$ satisfying $p \equiv a(\bmod q)$ up to $B$, and the above asymptotic follows from Dirichlet's theorem on primes in arithmetic progressions. Therefore we may find a constant $C_{2}$ such that

$$
\omega\left(\mathcal{D}^{\prime}\right)>\frac{C_{2} \xi_{4}}{\log \xi_{4}}
$$

for all $B$ sufficiently large. Observe that for a square-free number $l$, we have

$$
\sigma_{0}(l)=\prod_{p \mid l}(1+1)=2^{\omega(l)} .
$$

From the definition of $\mathcal{D}$ and $\mathcal{D}^{\prime}$, it follows that

$$
(3 / 2)^{\omega\left(\mathcal{D}^{\prime}\right)}<(3 / 2)^{\omega\left(D^{\prime}\right)} C\left(\xi_{1}\right)(3 / 2)^{\operatorname{gcd}\left(\mathcal{D}^{\prime}, u_{2}\right)}<(3 / 2)^{\omega(\mathcal{D})} C\left(\xi_{1}\right) \sigma_{0}\left(\operatorname{gcd}\left(\mathcal{D}^{\prime}, u_{2}\right) .\right.
$$

Hence, there exists a positive number $C_{3}$ such that

$$
\frac{1}{(3 / 2)^{\omega(\mathcal{D})}}<\frac{C_{3}}{(3 / 2)^{\omega\left(\mathcal{D}^{\prime}\right)}} \sigma_{0}\left(\operatorname{gcd}\left(\mathcal{D}^{\prime}, u_{2}\right)\right) \exp \left(2(k-1) \xi_{1}\right)
$$


From here we obtain the estimate

$$
N_{4}(B)=O\left(\exp \left(2(n+1)(k-1) \xi_{1}\right) \sum_{u_{2} \leq \xi_{3}} \frac{B^{n} \tau_{F}\left(u_{2}\right) \sigma_{-1 / 4}\left(u_{2}\right) \sigma_{0}\left(\operatorname{gcd}\left(\mathcal{D}^{\prime}, u_{2}\right)\right)}{(3 / 2)^{\omega\left(\mathcal{D}^{\prime}\right)} u_{2}}\right) .
$$

We now estimate the sum

$$
S(t)=\sum_{u_{2} \leq t} \tau_{F}\left(u_{2}\right) \sigma_{-1 / 4}\left(u_{2}\right) \sigma_{0}\left(\operatorname{gcd}\left(\mathcal{D}, u_{2}\right)\right)
$$

We proceed, as with Hooley, by invoking his Lemma 6.2 in [25]. We then have

$$
\begin{aligned}
S(t) & \leq \sum_{h \mid \mathcal{D}} \mu^{2}(h) \sigma_{0}(h) \sum_{\substack{u_{2} \leq t \\
u_{2} \equiv 0}} \tau_{F}\left(u_{2}\right) \sigma_{-1 / 4}\left(u_{2}\right) \\
& =\sum_{h \mid \mathcal{D}} \mu^{2}(h) \sigma_{0}(h) \sum_{\substack{u_{2}^{\prime} h \leq t \\
\operatorname{gcd}\left(u_{2}^{\prime}, h\right)=1}} \tau_{F}\left(h u_{2}^{\prime}\right) \sigma_{-1 / 4}\left(h u_{2}^{\prime}\right) \\
& \leq \sum_{h \mid \mathcal{D}} \mu^{2}(h) \sigma_{0}(h) \tau_{F}(h) \sigma_{-1 / 4}(h) \sum_{u_{2}^{\prime} \leq t / h} \tau_{F}\left(u_{2}^{\prime}\right) \sigma_{-1 / 4}\left(u_{2}^{\prime}\right) \\
& =O\left(t \sum_{h \mid \mathcal{D}} \frac{\mu^{2}(h) \sigma_{0}(h) \tau_{F}(h) \sigma_{-1 / 4}(h)}{h}\right) \\
& =O\left(t \prod_{w \leq \xi_{4}}\left(1+\frac{2 d+1}{w}\right)\right) \\
& =O\left(t\left(\log \xi_{4}\right)^{2 d+1}\right) \\
& =O\left(t(\log \log B)^{2 d+1}\right) .
\end{aligned}
$$

By following Hooley's treatment of the term $N^{(6)}(X)$ in Section 8 of his paper [25] and cutting the range of the summation in (5.18) into dyadic parts, we see that, for some positive number $C_{4}$ we have

$$
N_{4}(B)=O\left(\frac{B^{n} \exp \left(2(n+1)(k-1) \xi_{1}\right)}{(\log B)^{C_{4} / \log _{3} B}}\right) .
$$

We now put

$$
\xi_{1}(B)=\max \left\{1, \frac{C_{4} \log \log B}{4(n+1)(k-1) \log _{3} B}\right\}
$$

so that

$$
\frac{\exp (2(n+1)(k-1) g(B))}{\exp \left(C_{4} \log _{2} B / \log _{3} B\right)}=\exp \left(\frac{-C_{4} \log _{2} B}{2 \log _{3} B}\right)
$$

whence

$$
N_{4}(B)=O\left(B^{n} \exp \left(\frac{-C_{4} \log _{2} B}{2 \log _{3} B}\right)\right)=o\left(B^{n}\right)
$$


Next we turn our attention to the much more difficult component

$$
N_{5}(B)=\sum_{\substack{u_{1} \mid C\left(\xi_{1}\right) \\ u_{2} \leq \xi_{3}}} \frac{M_{\mathcal{R}_{l}}^{++}(B)}{k^{\omega(\mathcal{D})}}
$$

Recall from (5.12) that

$$
E_{\mathcal{R}_{l}}\left(t_{1}, \cdots, t_{n} ; l\right)=E_{\mathcal{R}_{u_{1}}} E_{\mathcal{R}_{u_{2}}} E_{\mathcal{R}_{\mathcal{D}}}
$$

The term $E_{\mathcal{R}_{u_{1}}}\left(t_{1}, \cdots, t_{n} ; u_{1}\right)$ can be trivially estimated by $u_{1}^{n}$, which is of size $O\left(\exp \left(\frac{C_{4} \log _{2} B}{4(k-1) \log _{3} B}\right)\right)$. We now consider the term $E_{\mathcal{R}_{u_{2}}}$. For each prime $p$ dividing $u_{2}$ we write

$$
F(\mathbf{x})=F^{*}(\mathbf{x}) \prod_{j=1}^{\tau(p)} \mathcal{L}_{i}(\mathbf{x})
$$

where $F^{*}$ and $\mathcal{L}_{i}$ have coefficients in $\mathbb{Z}_{p}$. We then write $E_{\mathcal{R}_{u_{2}}}$ as

$$
\begin{aligned}
E_{\mathcal{R}_{u_{2}}}\left(t_{1}, \cdots, t_{n} ; u_{2}\right) & =\prod_{p \mid u_{2}}\left(\sum_{\left.1 \leq i \leq \tau_{F}(p)\right)} \sum_{\substack{\mathcal{L}_{i}\left(a_{1}, \cdots, a_{n}\right) \equiv 0 \\
\left(a_{1}, \cdots, a_{n}\right) \in \mathbb{F}_{p}^{n} \\
(\bmod p)}} e^{2 \pi i\left(a_{1} t_{1}+\cdots+a_{n} t_{n}\right) / p}\right) \\
& =\prod_{p \mid u_{2}} S\left(t_{1}, \cdots, t_{n} ; p\right) .
\end{aligned}
$$

We shall obtain the following estimate for $S\left(t_{1}, \cdots, t_{n} ; p\right)$ :

Lemma 5.8. Let $p$ be a prime, and put

$$
S\left(t_{1}, \cdots, t_{n} ; p\right)=\sum_{1 \leq i \leq \tau_{F}(p)} \sum_{\substack{\left(a_{1}, \cdots, a_{n}\right) \in \mathbb{F}_{p}^{n} \\ \mathcal{L}_{i}\left(a_{1}, \cdots, a_{n}\right) \equiv 0 \quad(\bmod p)}} e^{2 \pi i\left(a_{1} t_{1}+\cdots+a_{n} t_{n}\right) / p} .
$$

Then we have

$$
S\left(t_{1}, \cdots, t_{n} ; p\right) \begin{cases}\leq \tau_{F}(p) p^{n-1}, & \text { if } t_{1} x_{1}+\cdots+t_{n} x_{n} \text { divides } F(\mathbf{x}) \text { over } \mathbb{F}_{p} \\ =0, & \text { otherwise. }\end{cases}
$$

Proof. We consider two scenarios. Suppose that

$$
\mathcal{L}_{s}\left(x_{1}, \cdots, x_{n}\right)=v_{1}^{(s)} x_{1}+\cdots+v_{n}^{(s)} x_{n}, v_{j}^{(s)} \in \mathbb{Z}_{p} \text { for } 1 \leq j \leq n .
$$

If $\left(t_{1}, \cdots, t_{n}\right) \equiv \lambda\left(v_{1}^{(s)}, v_{2}^{(s)}, \cdots, v_{n}^{(s)}\right)(\bmod p)$ for some $\lambda \in \mathbb{F}_{p}^{*}$, then

$$
\sum_{\substack{\left(a_{1}, \cdots, a_{n}\right) \in \mathbb{F}_{p}^{n} \\ \mathcal{L}_{s}\left(a_{1}, \cdots, a_{n}\right) \equiv 0 \quad(\bmod p)}} e^{2 \pi i\left(a_{1} t_{1}+\cdots+a_{n} t_{n}\right) / p}=p^{n-1} .
$$

Observe that since $p \nmid \Delta(F)$, that there does not exist $1 \leq s \leq \tau_{F}(p)$ such that $p \mid v_{j}^{(s)}$ for all $j=1, \cdots, n$. We may suppose, without loss of generality, that $v_{1}^{(s)} \not \equiv 0$ $(\bmod p)$. Suppose that $\mathbf{a} \in \mathbb{F}_{p}^{n}$ is such that

$$
\mathcal{L}_{s}(\mathbf{a}) \equiv 0 \quad(\bmod p) .
$$


It then follows that

$$
a_{1} \equiv-\left(v_{1}^{(s)}\right)^{-1}\left(v_{2}^{(s)} a_{2}+\cdots+v_{n}^{(s)} a_{n}\right)
$$

This implies

$$
\sum_{\substack{\left(a_{1}, \cdots, a_{n}\right) \in \mathbb{F}_{p}^{n} \\ \mathcal{L}_{s}\left(a_{1}, \cdots, a_{n}\right) \equiv 0}} e^{2 \pi i\left(a_{1} t_{1}+\cdots+a_{n} t_{n}\right) / p}=\sum_{\left(a_{2}, \cdots, a_{n}\right) \in \mathbb{F}_{p}^{n}} e^{2 \pi i\left(a_{2}\left(t_{2}-t_{1}\left(v_{1}^{(s)}\right)^{-1} v_{2}^{(s)}\right)+\cdots+a_{n}\left(t_{n}-t_{1}\left(v_{1}^{(s)}\right)^{-1} v_{n}^{(s)}\right)\right) / p} .
$$

The right hand side can be written as

$$
\prod_{j=2}^{n} \sum_{a_{j} \in \mathbb{F}_{p}} e^{2 \pi i a_{j}\left(v_{1}^{(s)} t_{j}-t_{1} v_{j}^{(s)}\right) / p}
$$

For each $j$, the sum

$$
\sum_{a_{j} \in \mathbb{F}_{p}} e^{2 \pi i a_{j}\left(v_{1}^{(s)} t_{j}-t_{1} v_{j}^{(s)}\right) / p}
$$

is zero unless the exponent is identically zero. This shows that (5.23) is non-zero if and only if $v_{1}^{(s)} t_{j} \equiv t_{1} v_{j}^{(s)}(\bmod p)$ for $j=2, \cdots, n$. This implies that

$$
\begin{aligned}
\left(t_{1}, \cdots, t_{n}\right) & \equiv t_{1}\left(v_{1}^{(s)}\right)^{-1}\left(v_{1}^{(s)}, v_{1}^{(s)} t_{2} t_{1}^{-1}, \cdots, v_{1}^{(s)} t_{n} t_{1}^{-1}\right) \quad(\bmod p) \\
& \equiv t_{1}\left(v_{1}^{(s)}\right)^{-1}\left(v_{1}^{(s)}, v_{2}^{(s)}, \cdots, v_{n}^{(j)}\right) \quad(\bmod p),
\end{aligned}
$$

hence the first situation is the only case where the sum

$$
\sum_{\substack{\left(a_{1}, \cdots, a_{n}\right) \in \mathbb{F}_{p}^{n} \\ \mathcal{L}_{s}\left(a_{1}, \cdots, a_{n}\right) \equiv 0 \quad(\bmod p)}} e^{2 \pi i\left(a_{1} t_{1}+\cdots+a_{n} t_{n}\right) / p}
$$

is non-zero. In other words, we have

$$
S\left(t_{1}, \cdots, t_{n} ; p\right) \begin{cases}\leq \tau_{F}(p) p^{n-1}, & \text { if } t_{1} x_{1}+\cdots+t_{n} x_{n} \text { divides } F(\mathbf{x}) \text { over } \mathbb{F}_{p}, \\ =0, & \text { otherwise },\end{cases}
$$

as desired.

For square-free $l$, let us write

$$
S\left(t_{1}, \cdots, t_{n} ; l\right)=\prod_{p \mid l} S\left(t_{1}, \cdots, t_{n} ; p\right) .
$$

We have the following lemma:

Lemma 5.9. Let $u_{1}, u_{2}, \mathcal{D}$ be as in Lemma 5.6. Then

$$
\sum_{\substack{u_{1} \mid C\left(\xi_{1}\right) \\ u_{2} \leq \xi_{3}}} \frac{M_{l}^{++}(B)}{k^{\omega(\mathcal{D})}}=O\left(\exp \left(2(n+1)(k-1) \xi_{1}\right) \sum_{u_{2} \leq \xi_{3}} \frac{B^{n}}{u_{2}^{n}} \sum_{\left|t_{1}\right|, \cdots,\left|t_{n}\right| \leq l / B}^{\prime} S\left(t_{1}, \cdots, t_{n} ; u_{2}\right)\right)
$$


Proof. Recall that

$$
M_{l}^{++}(B)=\frac{B^{n}}{u_{1}^{n} u_{2}^{n} \mathcal{D}^{n}} \sum_{\left|t_{i}\right| \leq l / B}^{\prime}\left|E_{\mathcal{R}_{l}}\left(t_{1}, \cdots, t_{n} ; l\right)\right| .
$$

Note that

$$
\left|E_{\mathcal{R}_{l}}\left(t_{1}, \cdots, t_{n} ; \mathcal{D}\right)\right|=O\left(\mathcal{D}^{n} \sigma_{-1 / 4}(\mathcal{D})\right)
$$

and the multiplicativity of $E_{\mathcal{R}_{l}}$ implies that

$$
\left|E_{\mathcal{R}_{l}}\left(t_{1}, \cdots, t_{n} ; l\right)\right|=O\left(u_{1}^{n} \mathcal{D}^{n} \sigma_{-1 / 4}(\mathcal{D}) S\left(t_{1}, \cdots, t_{n} ; u_{2}\right)\right) .
$$

Next note that

$$
\sigma_{-1 / 4}(\mathcal{D})=O\left(k^{\omega(\mathcal{D})}\right)
$$

since $k \geq 2$. This then implies (5.24), since the number of divisors of $C\left(\xi_{1}\right)$ does not exceed $C\left(\xi_{1}\right)$.

We now assess $S\left(t_{1}, \cdots, t_{n} ; u_{2}\right)$ for an $n$-tuple $\left(t_{1}, \cdots, t_{n}\right) \in \mathbb{Z}^{n}$. By Lemma 5.8 , this is zero unless for each prime $p \mid u_{2}$ there exists $\lambda_{p} \in \mathbb{F}_{p}$ and $1 \leq s_{p} \leq \tau_{F}(p)$ such that $\left(t_{1}, \cdots, t_{n}\right) \equiv \lambda_{p}\left(v_{1}^{\left(s_{p}\right)}, v_{2}^{\left(s_{p}\right)}, \cdots, v_{n}^{\left(s_{p}\right)}\right)(\bmod p)$. One checks at once that for a fixed vector $\boldsymbol{v}=\left(v_{1}, \cdots, v_{n}\right)$, the set

$$
\left\{\left(x_{1}, \cdots, x_{n}\right) \in \mathbb{Z}^{n}:\left(x_{1}, \cdots, x_{n}\right) \equiv \lambda\left(v_{1}, \cdots, v_{n}\right) \quad(\bmod p) \text { for some } \lambda \in \mathbb{F}_{p}\right\}
$$

is a lattice. For each prime $p$ dividing $u_{2}$, there are $\tau_{F}(p) \leq d$ such lattices to consider. If $\left(t_{1}, \cdots, t_{n}\right) \in \mathbb{Z}^{n}$ is such that $S\left(t_{1}, \cdots, t_{n} ; u_{2}\right)$ is non-zero, then it must lie on one such lattice for each prime divisor of $u_{2}$. Therefore, $\left(t_{1}, \cdots, t_{n}\right)$ lies on one of at most $d^{\omega\left(u_{2}\right)}$ lattices, each with determinant $u_{2}^{n-1}$. Let $\mathfrak{L}\left(u_{2}\right)$ denote the set of lattices for which the $n$-tuples $\left(t_{1}, \cdots, t_{n}\right)$ such that $S\left(t_{1}, \cdots, t_{n} ; u_{2}\right) \neq 0$ are restricted to.

We now replace the bound $l / B$ for the variables $t_{i}$ in Lemma 5.24 by something that is easier to work with. Observe that

$$
u_{1} \mathcal{D}=O\left(\exp \left(2(k-1) \xi_{1}\right)\left(\frac{B^{2}}{u_{2}}\right)^{1 / 11}\right) \text {. }
$$

Therefore, it follows that

$$
\begin{gathered}
\frac{l}{B}=\frac{u_{1} u_{2} \mathcal{D}}{B}=O\left(\exp \left(2(k-1) \xi_{1}\right) \frac{B^{2 / 11}}{u_{2}^{1 / 11}} \frac{u_{2}}{B}\right) \\
=O\left(\exp \left(2(k-1) \xi_{1}\right)\left(\frac{u_{2}^{10 / 11}}{B^{9 / 11}}\right)\right) .
\end{gathered}
$$

Moreover, we have

$$
\exp \left(2(k-1) \xi_{1}\right) \frac{u_{2}^{10 / 11}}{B^{9 / 11}}=O\left(\frac{u_{2}^{9 / 10}}{B^{4 / 5}}\right)
$$

since

$$
\frac{u_{2}^{9 / 10}}{B^{4 / 5}} \cdot \frac{B^{9 / 11}}{u_{2}^{10 / 11}}=\left(\frac{B^{2}}{u_{2}}\right)^{1 / 55} \gg(\log B)^{\frac{2 k}{165}} \gg(\log B)^{\frac{C_{4}}{2(n+1) \log _{3} B}}
$$


Put

$$
Q(B)=\sum_{u_{2} \leq \xi_{3}} \frac{1}{u_{2}^{n}} \sum_{\left|t_{1}\right|, \cdots,\left|t_{n}\right| \leq u_{2}^{9 / 10} / B^{4 / 5}}^{\prime} S\left(t_{1}, \cdots, t_{n} ; u_{2}\right) .
$$

Then it is clear that

$$
\sum_{\substack{u_{1} \mid C\left(\xi_{1}\right) \\ u_{2} \leq \xi_{3}}} \frac{M_{l}^{++}(B)}{k^{\omega(\mathcal{D})}}=O\left(B^{n} g(B)^{k-1} Q(B)\right) .
$$

We shall assess $Q(B)$ by restricting the range of $u_{2}$ to a dyadic interval of the form $(U / 2, U]$, with $U \leq \xi_{3}$. Denote this contribution to $Q(B)$ by $Q_{U}(B)$. We have the following lemma:

Lemma 5.10. Let $Q(B)$ be as in (5.27). Then there exists a positive number $C_{5}$ such that for all $U>1$, we have

$$
Q_{U}(B)=O\left(\frac{U^{9 / 10}(\log B)^{C_{5}}}{B^{8 / 5}}\right) .
$$

Proof. Let us write $F_{s}\left(x_{1}, x_{s}\right)$ for the product

$$
F_{s}\left(x_{1}, x_{s}\right)=\prod_{j=1}^{d}\left(\psi_{1}^{(j)} x_{1}+\psi_{s}^{(j)} x_{s}\right),
$$

where $\psi_{s}^{(j)}$ are as in (4.1). Note that each $F_{s}$ has integer coefficients. Moreover, since $F$ is irreducible over $\mathbb{Q}$ it follows that each $F_{s}$ is a perfect power of a binary form with integer coefficients. Further, $F_{s}$ is not identically zero for $s=2, \cdots, n$. If we fix a vector $\left(t_{1}, \cdots, t_{n}\right) \in \mathbb{Z}^{n}$, then there are only at most $\sigma_{0}\left(F_{2}\left(t_{2},-t_{1}\right)\right)$ many $u_{2}$ such that $\left(t_{1}, \cdots, t_{n}\right) \in \Lambda$ for some $\Lambda \in \mathfrak{L}\left(u_{2}\right)$. To see this, if $\left(t_{1}, \cdots, t_{n}\right) \in \Lambda$ for $\Lambda \in \mathfrak{L}\left(u_{2}\right)$, then for each prime $p \mid u_{2}$, we have $\left(t_{1}, \cdots, t_{n}\right) \equiv \lambda_{p}\left(1, v_{2}^{(s)}, \cdots, v_{n}^{(s)}\right)(\bmod p)$ for some $\lambda_{p} \in \mathbb{F}_{p}$ and $1 \leq s \leq \tau_{F}(p)$. Then it follows that $t_{2} \equiv t_{1} v_{2}^{(s)}(\bmod p)$, hence it follows that

$$
F_{2}\left(t_{2},-t_{1}\right) \equiv 0 \quad(\bmod p) \text {. }
$$

This implies that $u_{2} \mid F_{2}\left(t_{2},-t_{1}\right)$, as claimed. Further, by the same argument we get that $u_{2} \mid F_{s}\left(t_{s},-t_{1}\right)$ for all $2 \leq s \leq n$.

Now we can estimate $Q_{U}(B)$ when $U$ is suitably small as follows:

$$
\begin{aligned}
Q_{U}(B) & \leq \frac{2^{n}}{U^{n}} \sum_{U / 2<u_{2} \leq U} \sum_{\left|t_{1}\right|, \cdots,\left|t_{n}\right| \leq U^{9 / 10} / B^{4 / 5}}^{\prime} S\left(t_{1}, \cdots, t_{n} ; u_{2}\right) \\
& \leq \frac{2^{n}}{U} \sum_{U / 2<u_{2} \leq U} d^{\omega\left(u_{2}\right)} \sum_{\substack{\left|t_{1}\right|, \cdots,\left|t_{n}\right| \leq U^{9 / 10} / B^{4 / 5} \\
u_{2} \mid \operatorname{gcd}\left(F_{2}\left(t_{2},-t_{1}\right), \cdots, F_{n}\left(t_{n},-t_{1}\right)\right)}}^{\prime} 1 .
\end{aligned}
$$

Observe that when $t_{1}, t_{2}$ are fixed, then the condition $u_{2} \mid F_{j}\left(t_{j},-t_{1}\right)$ constrains each $t_{j}, j=3, \cdots, n$ to at most $d^{\omega\left(u_{2}\right)}$ congruence classes modulo $u_{2}$, and for each congruence class, at most $\left(2 U^{9 / 10} B^{-4 / 5}\right) / u_{2}+1$ choices in the range $\left[-U^{9 / 10} / B^{4 / 5}, U^{9 / 10} / B^{4 / 5}\right]$. 
Since $U / 2<u_{2} \leq U$, there is at most one choice when $B$ is sufficiently large. By the binomial theorem, for a number $A$ and a square-free positive integer $m$, we have

$$
\sum_{r \mid m} A^{\omega(r)}=(A+1)^{\omega(m)}
$$

By permuting the variables if necessary, we may assume that $t_{1} \neq 0$, at the cost of a factor of $n$. Hence

$$
\begin{aligned}
Q_{U}(B) & \leq \frac{n 2^{n}}{U} \sum_{\substack{\left|t_{1}\right|,\left|t_{2}\right| \leq U^{9 / 10} / B^{4 / 5} \\
t_{1} \neq 0}} \sum_{u_{2} \mid F_{2}\left(t_{2},-t_{1}\right)} d^{(n-1) \omega\left(u_{2}\right)} \\
& =\frac{n 2^{n}}{U} \sum_{\substack{\left|t_{1}\right|,\left|t_{2}\right| \leq U^{9 / 10} / B^{4 / 5} \\
t_{1} \neq 0}}^{\prime}\left(d^{n-1}+1\right)^{\omega\left(F_{2}\left(t_{2},-t_{1}\right)\right)},
\end{aligned}
$$

so by Lemma 10.1 in [25], there exists a positive number $C_{5}$ such that

$$
Q_{U}(B)=O\left(\frac{U^{9 / 10}(\log B)^{C_{5}}}{B^{8 / 5}}\right) .
$$

If $U$ is relatively small, say $U<B^{5 / 3}$, then this is a satisfactory bound. Otherwise, we use Lemma 10.2 in [25], which we state as

Lemma 5.11. (Hooley, 2009) Set $\Xi(B)=B^{\frac{1}{6(\log \log B)^{2}}}$. Fix $u_{2} \leq \xi_{3}$. Let $\omega^{\dagger}(m)$ denote the number of distinct prime factors of $m$ that exceed $\Xi$ and let

$$
l^{*}=\prod_{\substack{p \leq \Xi \\ p \mid u_{2}}} p
$$

and

$$
l^{\dagger}=\prod_{\substack{p>\Xi \\ p \mid u_{2}}} p .
$$

Suppose that $l^{*} \leq B^{1 / 6}$. Then, for any positive constant $C_{6}$ and for $B^{1 / 2}<Y<B$, there exists a positive number $C_{7}$, depending only on $C_{6}$, such that

$$
\sum_{\substack{\left(u_{1}, u_{2}\right) \equiv\left(t_{1}, t_{2}\right) \\\left|u_{1},\right| u_{2} \mid \leq Y}}^{\prime} C_{6}^{\omega^{\dagger}\left(F\left(u_{1}, u_{2}\right)\right)}=O\left(\frac{Y^{2}(\log \log B)^{C_{7}}}{\left(l^{*}\right)^{2}}\right) .
$$

When $U>B^{5 / 3}$ we employ the divisors $l^{*}, l^{\dagger}$ of $u_{2}$ as in Lemma 5.11. Suppose firstly that $l^{*}>B^{1 / 6}$. This means that

$$
B^{1 / 6}<\Xi^{\omega\left(l^{*}\right)} \leq \Xi^{\omega\left(u_{2}\right)}
$$

which shows that

$$
\omega\left(u_{2}\right)>\left(\log _{2} B\right)^{2} .
$$


Hence, either $\omega\left(u_{2}\right)>\left(\log _{2} B\right)^{2}$ or $l^{*} \leq B^{1 / 6}$. Put

$$
Q_{U}^{(1)}(B)=\sum_{\substack{U / 2<u_{2} \leq U \\ \omega\left(u_{2}\right)>\left(\log _{2} B\right)^{2}}} \frac{1}{u_{2}^{n}} \sum_{\left|t_{1}\right|, \cdots,\left|t_{n}\right| \leq u_{2}^{9 / 10} / B^{4 / 5}}^{\prime} S\left(t_{1}, \cdots, t_{n} ; u_{2}\right)
$$

and

$$
Q_{U}^{(2)}(B)=\sum_{\substack{U / 2<u_{2} \leq U \\ l^{*} \leq B^{1 / 6}}} \frac{1}{u_{2}^{n}} \sum_{\left|t_{1}\right|, \cdots,\left|t_{n}\right| \leq u_{2}^{9 / 10} / B^{4 / 5}}^{\prime} S\left(t_{1}, \cdots, t_{n} ; u_{2}\right) .
$$

We have the following estimates for $Q_{U}^{(1)}(B)$ and $Q_{U}^{(2)}(B)$ :

Lemma 5.12. Let $Q_{U}^{(1)}(B), Q_{U}^{(2)}(B)$ be as in (5.30) and (5.31) respectively. Then there exists a positive number $C_{6}$ depending only on $d, n$ such that

$$
Q_{U}^{(1)}(B)=O_{d}\left(\frac{U^{4 / 5}(\log B)^{C_{6}}}{B^{8 / 5}(\log B)^{\log _{2} B}}\right)
$$

and

$$
Q_{U}^{(2)}(B)=O_{d}\left(\frac{U^{4 / 5} \log B\left(\log _{2} B\right)^{C_{7}}}{B^{8 / 5}}\right) .
$$

Proof. To estimate $Q_{U}^{(1)}(B)$, by (5.29) we have

$$
\begin{aligned}
Q_{U}^{(1)}(B) & \leq \frac{n 2^{n}}{U} \sum_{\substack{u_{2} \leq U \\
\omega\left(u_{2}\right)>(\log \log B)^{2}}} \sum_{\left|t_{1}\right|,\left|t_{2}\right| \leq u_{2}^{9 / 10} / B^{4 / 5}}^{\prime} d^{(n-1) \omega\left(F\left(t_{2},-t_{1}\right)\right)} \\
& \ll_{n} \frac{1}{U} \sum_{\left|t_{1}\right|,\left|t_{2}\right| \leq U^{9 / 10} / B^{4 / 5}}^{\prime} \sum_{\substack{u_{2} \mid F_{2}\left(t_{2},-t_{1}\right) \\
\omega\left(u_{2}\right)>\left(\log _{2} B\right)^{2}}} d^{(n-1) \omega\left(u_{2}\right)} .
\end{aligned}
$$

Observe that since $u_{2} \mid F_{2}\left(t_{2},-t_{1}\right)$, we have

$$
d^{(n-1) \omega\left(u_{2}\right)}=\frac{d^{(n-1) \omega\left(u_{2}\right)} e^{\left(\log _{2} B\right)^{2}}}{(\log B)^{\log _{2} B}}<\frac{\left(3 d^{n-1}\right)^{\omega\left(u_{2}\right)}}{(\log B)^{\log _{2} B}} .
$$

By the binomial theorem and the fact that $u_{2}$ is square-free, it follows that

$$
\sum_{\substack{u_{2} \mid F_{2}\left(t_{2},-t_{1}\right) \\ \omega\left(u_{2}\right)>\left(\log _{2} B\right)^{2}}} d^{(n-1) \omega\left(u_{2}\right)} \leq \sum_{\substack{u_{2} \mid F_{2}\left(t_{2},-t_{1}\right) \\ \omega\left(u_{2}\right)>\left(\log _{2} B\right)^{2}}} \frac{\left(3 d^{n-1}\right)^{\omega\left(u_{2}\right)}}{(\log B)^{\log _{2} B}}=\frac{\left(3 d^{n-1}+1\right)^{\omega\left(F_{2}\left(t_{2},-t_{1}\right)\right)}}{(\log B)^{\log _{2} B}} .
$$

Hence, we see that for some positive $C_{6}$

$$
\begin{aligned}
Q_{U}^{(1)}(B) & \ll_{n} \frac{1}{U(\log B)^{\log _{2} B}} \sum_{\left|t_{1}\right|,\left|t_{2}\right| \leq U^{9 / 10} / B^{4 / 5}}^{\prime}\left(3 d^{n-1}+1\right)^{\omega\left(F_{2}\left(t_{2},-t_{1}\right)\right)} \\
& =O\left(\frac{U^{4 / 5}(\log B)^{C_{6}}}{B^{8 / 5}(\log B)^{\log _{2} B}}\right)
\end{aligned}
$$

by Lemma 10.1 in [25] again. This completes the estimation of $Q_{U}^{(1)}(B)$. 
Observe that

$$
\frac{U^{4 / 5}}{B^{8 / 5}}=O\left(\xi_{3}^{4 / 5} B^{-8 / 5}\right)=O\left((\log B)^{-8 k / 15}\right),
$$

and thus the desired conclusion for $Q_{U}^{(1)}(B)$ holds.

The sum $Q_{U}^{(2)}(B)$ is more difficult. The key tool will be Lemma 5.11. Recall that $Q_{U}^{(2)}(B)$ consists of the contribution from those tuples for which $l^{*} \leq B^{1 / 6}$ and $U>B^{5 / 3}$. By the multiplicativity of $S\left(t_{1}, \cdots, t_{n}, \cdot\right)$, it follows that

$$
Q_{U}^{(2)}(B) \leq \frac{2^{n}}{U^{n}} \sum_{\left|t_{1}\right|, \cdots,\left|t_{n}\right| \leq U^{9 / 10} / B^{4 / 5}}^{\prime} \sum_{\substack{l^{*} l^{\dagger} \leq U \\ l^{*} \leq B^{1 / 6}}} S\left(t_{1}, \cdots, t_{n} ; l^{*}\right) S\left(t_{1}, \cdots, t_{n} ; l^{\dagger}\right) .
$$

We rearrange the summation to obtain

$$
\frac{2^{n}}{U^{n}} \sum_{\substack{l^{*} l^{\dagger} \leq U \\ l^{*} \leq B^{1 / 6}}} \sum_{\substack{b_{1}, \cdots, b_{n} \\\left(\bmod l^{*}\right)}} S\left(b_{1}, \cdots, b_{n} ; l^{*}\right) \sum_{\substack{\left|t_{1}\right|, \cdots,\left|t_{n}\right| \leq U^{9 / 10} / B^{4 / 5} \\ t_{i}=b_{i}\left(\bmod l^{*}\right)}}^{\prime} S\left(t_{1}, \cdots, t_{n} ; l^{\dagger}\right) .
$$

We estimate $S\left(t_{1}, \cdots, t_{n} ; l^{\dagger}\right)$ by $d^{\omega\left(l^{\dagger}\right)}\left(l^{\dagger}\right)^{n-1}$ when it is non-zero. Next we observe that from the proof of Lemma 5.10 that $S\left(t_{1}, \cdots, t_{n} ; l^{\dagger}\right)$ is non-zero only if $l^{\dagger}$ divides $F_{s}\left(t_{s},-t_{1}\right)$ for $s=2, \cdots, n$. Since $U>B^{5 / 3}$ and $l^{*} \leq B^{1 / 6}$, it follows that $l^{\dagger}>B^{3 / 2}$. Therefore $\left(U^{9 / 10} B^{-4 / 5}\right) / l^{\dagger} \ll B^{-1 / 2}(\log B)^{-2 k / 3}$. In other words, for sufficiently large $B$ and for fixed $t_{1}, t_{2}$, the congruence condition imposed by $l^{\dagger}$ leads to at most $d^{\omega\left(l^{\dagger}\right)}$ choices for $t_{3}, \cdots, t_{n}$ as before. It then follows that

$$
\begin{aligned}
& Q_{U}^{(2)}(B) \leq \frac{n 2^{n}}{U^{n}} \sum_{\substack{l^{*} \leq B^{1 / 6} \\
b_{1}, \cdots, b_{n}}} S\left(b_{1}, \cdots, b_{n} ; l^{*}\right) \sum_{\substack{\left.\left|t_{1}\right|,\left|t_{2}\right| \leq U^{9 / 10} / B^{*}\right) \\
t_{i} \equiv b_{i}}} \sum_{\left(\bmod l^{*}\right)} \sum_{l^{\dagger} \mid F_{2}\left(t_{2},-t_{1}\right)} d^{\omega\left(l^{\dagger}\right)}\left(l^{\dagger}\right)^{n-1} \\
& \leq \frac{n 2^{n}}{U} \sum_{\substack{l^{*} \leq B^{1 / 6} \\
b_{1}, \cdots, b_{n}}} \frac{S\left(t_{1}, \cdots, t_{n} ; l^{*}\right)}{\left(l^{*}\right)^{n-1}} \sum_{\substack{\left|t_{1}\right|,\left|t_{2}\right| \leq U^{9 / 10} / B^{* / 5} \\
t_{i} \equiv b_{i}\left(\bmod l^{*}\right)}}^{\prime} \sum_{l^{\dagger} \mid F_{2}\left(t_{2},-t_{1}\right)} d^{\omega\left(l^{\dagger}\right)} \\
& \ll \frac{n 2^{n}}{U} \sum_{\substack{l^{*} \leq B^{1 / 6} \\
b_{1}, \cdots, b_{n}}} \frac{S\left(b_{1}, \cdots, b_{n} ; l^{*}\right)}{\left(l^{*}\right)^{n-1}} \sum_{\substack{\left|t_{1}\right|,\left|t_{2}\right| \leq U^{9 / 10} / B^{4 / 5} \\
t_{i} \equiv b_{i}\left(\bmod l^{*}\right)}}^{\prime}\left(d^{n-1}+1\right)^{\omega^{\dagger}\left(F_{2}\left(t_{2},-t_{1}\right)\right)} \\
& \ll \frac{n 2^{n}}{U} \sum_{\substack{l^{*} \leq B^{1 / 6} \\
b_{1}, \cdots, b_{n}\left(\bmod l^{*}\right)}} \frac{S\left(b_{1}, \cdots, b_{n} ; l^{*}\right)}{\left(l^{*}\right)^{n-1}} \sum_{\substack{\left|t_{1}\right|,\left|t_{2}\right| \leq U^{9 / 10} / B^{4 / 5} \\
t_{1} \equiv b_{1}\left(\bmod l^{*}\right) \\
t_{2} \equiv b_{2} \quad\left(\bmod l^{*}\right)}}^{\prime}\left(d^{n-1}+1\right)^{\omega^{\dagger}\left(F\left(t_{2},-t_{1}\right)\right)} .
\end{aligned}
$$

Note that $U<\xi_{3}=C_{1} B^{2}(\log B)^{-2 k / 3}$, whence $U^{9 / 10} / B^{4 / 5}<B$. Further our assumption of $U>B^{5 / 3}$ shows that $U^{9 / 10} / B^{4 / 5}>B^{7 / 10}$. Hence, the innermost sum is treatable by Lemma 5.11. We then have

$$
Q_{U}^{(2)}(B)=O_{d}\left(\frac{U^{4 / 5}\left(\log _{2} B\right)^{C_{7}}}{B^{8 / 5}} \sum_{l^{*} \leq B^{1 / 6}} \frac{1}{\left(l^{*}\right)^{n+1}} \sum_{b_{1}, \cdots, b_{n}} S\left(b_{1}, \cdots, b_{n} ; l^{*}\right)\right)
$$


By the proof of Lemma 5.8 , we se that for each prime $p$ we have

$$
\sum_{b_{1}, \cdots, b_{n}} S\left(b_{1}, \cdots, b_{n} ; p\right)=p \cdot \tau_{F}(p) p^{n-1}=\tau_{F}(p) p^{n} .
$$

It thus follows from multiplicativity that for any squarefree $l$ we have

$$
\sum_{b_{1}, \cdots, b_{n}} S\left(b_{1}, \cdots, b_{n} ; l\right)=\tau_{F}(l) l^{n} .
$$

We then deduce that

$$
\sum_{l^{*} \leq B^{1 / 6}} \frac{1}{\left(l^{*}\right)^{n+1}} \sum_{b_{1}, \cdots, b_{n}} S\left(b_{1}, \cdots, b_{n} ; l^{*}\right) \leq \frac{\tau_{F}\left(l^{*}\right)}{l^{*}} .
$$

By Lemma 6.1 in [25], we then see that

$$
\begin{aligned}
Q_{U}^{(2)}(B) & =O_{d}\left(\frac{U^{4 / 5}\left(\log _{2} B\right)^{C_{7}}}{B^{8 / 5}} \sum_{l^{*} \leq B^{1 / 6}} \frac{\tau_{F}\left(l^{*}\right)}{l^{*}}\right) \\
& =O_{d}\left(\frac{U^{4 / 5}\left(\log _{2} B\right)^{C_{7}}}{B^{8 / 5}} \prod_{p \leq B^{1 / 6}}\left(1+\frac{\tau_{F}(p)}{p}\right)\right) \\
& =O_{d}\left(\frac{U^{4 / 5} \log B\left(\log _{2} B\right)^{C_{7}}}{B^{8 / 5}}\right)
\end{aligned}
$$

as desired.

By summing over $Q_{U}(B), Q_{U}^{(1)}(B), Q_{U}^{(2)}(B)$ over dyadic ranges of $U$ up to $\xi_{2}$, we then see that

$$
\begin{aligned}
& \sum_{1 \leq k \ll \log B} O_{d}\left(\frac{\left(B^{5 / 3} / 2^{k}\right)^{9 / 10}(\log B)^{C_{5}}}{B^{8 / 5}}\right)=O\left(\frac{(\log B)^{C_{5}}}{B^{1 / 10}}\right), \\
& \sum_{1 \leq k \ll \log B} O_{d}\left(\frac{\left(\xi_{3} / 2^{k}\right)^{4 / 5}(\log B)^{C_{6}}}{B^{8 / 5}(\log B)^{\log _{2} B}}\right)=O\left((\log B)^{C_{6}-8 k / 15-\log _{2} B}\right),
\end{aligned}
$$

and

$$
\sum_{1 \leq k \ll \log B} O_{d}\left(\frac{\left(\xi_{3} / 2^{k}\right)^{4 / 5} \log B\left(\log _{2} B\right)^{C_{7}}}{B^{8 / 5}}\right)=O_{d}\left(\frac{\left(\log _{2} B\right)^{C_{7}}}{(\log B)^{(2 k-3) / 3}}\right) .
$$

This shows that

$$
Q(B)=O_{F}\left(\frac{\left(\log _{2} B\right)^{C_{7}}}{(\log B)^{(2 k-3) / 3}}\right)=o(1),
$$

and by (5.28), (5.21), Lemma 4.2 and Lemma 5.6 we see that

$$
N_{3}(B)=o\left(B^{n}\right) \text {, }
$$

and this completes the proof of Theorem 1.1 . 


\section{REFERENCES}

[1] M. Bhargava, The geometric sieve and the density of squarefree values of invariant polynomials, arXiv, http://arxiv.org/abs/1402.0031, retrieved 08 Jul 2014.

[2] M. Bhargava, A. Shankar, Binary quartic forms having bounded invariants, and the boundedness of the average rank of elliptic curves, Annals of Mathematics 181 (2015), 191-242.

[3] M. Bhargava, A. Shankar, X. Wang, Squarefree values of polynomial discriminants I, arXiv:1611.09806 [math.NT] https://arxiv.org/abs/1611.09806

[4] T. D. Browning, Quantitative Arithmetic on Projective Varieties, Birkhauser, 2009.

[5] T. D. Browning, Power-free values of polynomials, Arch. Math. (2) 96 (2011), 139-150.

[6] D. Cox, J. Little, D. O'Shea, Ideals, Varieties, and Algorithms - An Introduction to Computational Algebraic Geometry and Commutative Algebra, Springer-Verlag, New York.

[7] T. Ekedahl, An infinite version of the Chinese remainder theorem, Comment. Math. Univ. St. Paul. 40 (1991), 53-59.

[8] P. Erdős, Arithmetical properties of polynomials, J. London Math. Soc. 28 (1953), 416-425.

[9] P. Erdös, K. Mahler, On the number of integers which can be represented by a binary form, J. London Math. Soc. 13 (1938), 134-139.

[10] T. Estermann, Einige Satze uber quadratfeie Zahlen, Math. Ann., 105 (1931), 653-662.

[11] J-H. Evertse, The number of solutions of decomposable form equations, Inventiones Mathematicae 122 (1995), 559-601.

[12] M. Filaseta, Powerfree values of binary forms, Journal of Number Theory 49 (1994), 250-268.

[13] F. Gouvea, B. Mazur, The square-free sieve and the rank of elliptic curves, Journal of the American Mathematical Society, 4 (1991), 1-23.

[14] A. Granville, $A B C$ allows us to count squarefrees, International Mathematics Research Notices, 9 (1998).

[15] G. Greaves, Power-free values of binary forms, Q. J. Math, (2) 43 (1992), 45-65.

[16] G.H. Hardy, E. M. Wright, An Introduction to the Theory of Numbers, 5th edition, Oxford University Press, London and New York, 1979.

[17] H. Halberstam, K. F. Roth, On the gaps between consecutive $k$-free integers, J. London Math. Soc., (2) 26 (1951), 268-273.

[18] D. R. Heath-Brown, Diophantine approximation with square free numbers, Math. Z., 187 (1984), 335-344.

[19] D. R. Heath-Brown, The density of rational points on curves and surfaces, The Annals of Mathematics (2) 155 (2002), 553-598.

[20] D. R. Heath-Brown, Counting rational points on algebraic varieties, Analytic number theory, 5195, Lecture Notes in Math., 1891, Springer, Berlin, 2006.

[21] D. R. Heath-Brown, Powerfree values of polynomials, Q. J. Math, (2) 64 (2013), 177-188.

$[22]$ H. A. Helfgott, Power-free values, large deviations, and integer points on irrational curves, Journal de Theorie des Nombres de Bordeaux, 19 (2007), 433-472.

[23] H. A. Helfgott, Square-free values of $f(p), f$ cubic, Acta Math., 213 (2014), 107-135.

[24] C. Hooley, On the power free values of polynomials, Mathematika 14 (1967), 21-26.

[25] C. Hooley, On the power-free values of polynomials in two variables, Analytic Number Theory: Essays in Honour of Klaus Roth (2009).

[26] C. Hooley, On the power-free values of polynomials in two variables II, Journal of Number Theory, 129 (2009), 1443-1455.

[27] M. N. Huxley, M. Nair, Power free values of polynomials III, Proc. London Math. Soc. 41 (1980), 66-82.

[28] S. Lang, A. Weil, Number of points of varieties over finite fields, American Journal of Mathematics, (4) $\mathbf{7 6}$ (1954), 819-827.

[29] J. Maynard, Primes represented by incomplete norm forms, arXiv:1507.05080 [math.NT], http://arxiv.org/abs/1507.05080.

[30] M. Nair, Power free values of polynomials, Mathematika, 23 (1976), 159-183.

[31] M. Nair, Power free values of polynomials II, Proc. London. Math. Soc. (3) 38 (1979), 353-368. 
[32] B. Poonen, Squarefree values of multivariate polynomials, Duke Math. J., (2) 118 (2003), 353373.

[33] T. Reuss, Power-free values of polynomials, preprint.

[34] G. Ricci, Riecenche aritmetiche sui polynomials, Rend. Circ. Mat. Palermo 57 (1933), 433-475.

[35] J. B. Rosser, L. Schoenfeld, Approximate formulas for some functions of prime numbers, Illinois J. Math., (1) 6 (1962), 64-94.

[36] W. M. Schmidt, The number of solutions of norm form equations, Transactions of the American Mathematical Society, (1) 317 (1990), 197-227.

[37] C. L. Stewart, On the number of solutions of polynomial congruences and Thue equations, Journal of the American Mathematical Society, (4) 4 (1991), 793-835.

[38] C. L. Stewart, J. Top, On the ranks of twists of elliptic curves and power-free values of binary forms, Journal of the American Mathematical Society, (4) 8 (1995), 943-973.

[39] A. Thue, Uber Annaherungswerte algebraischer Zahlen, Journal fur die reine und angewandte Mathematik, 135 (1909), 284-305.

[40] J. D. Vaaler, Some extremal functions in Fourier analysis, Bulletin of the AMS, (2) 12 (1985), 183-216.

[41] S. Y. Xiao, Power-free values of binary forms and the global determinant method, Int Math Res Notices (2016) doi: 10.1093/imrn/rnw165, First published online: July 27, 2016.

Mathematical Institute, University of Oxford, Oxford, UK 